\title{
DIRICHLET EIGENVALUES OF CONES IN THE SMALL APERTURE LIMIT
}

\author{
THOMAS OURMIÈRES-BONAFOS*
}

\begin{abstract}
We are interested in finite cones of fixed height 1 parametrized by their opening angle. We study the eigenpairs of the Dirichlet Laplacian in such domains when their apertures tend to 0 . We provide multi-scale asymptotics for eigenpairs associated with the lowest eigenvalues of each fiber of the Dirichlet Laplacian. In order to do this, we investigate the family of their one-dimensional Born-Oppenheimer approximations. The eigenvalue asymptotics involves powers of the cube root of the aperture, while the eigenfunctions include simultaneously two scales.
\end{abstract}

\section{Introduction}

\subsection{Motivations and related questions}

Finding an explicit expression of the first Dirichlet eigenvalues in two or three dimensional domains is not an easy task, in general. We know how to proceed when the domain is a product reducing the problem to solving ordinary differential equations. Nevertheless, even for simple two dimensional domains like triangles this question is still complicated. This specific question is detailed in [17] where a finite term asymptotics is provided in the regime $\theta$ goes to 0 (where $\theta$ is the aperture of the triangle). More recently [11] gives a complete asymptotics for right-angled triangles.

In the latter paper, the aim of the authors was the study of a broken waveguide with corner in the small angle regime. The knowledge on triangles with small aperture leads to a comparison between the waveguide and a triangle. The question of waveguides with corners has already been investigated for the L-shape waveguide in [15]. For an arbitrary angle [3] provides an asymptotics of the lowest eigenvalues when the angle goes to $\pi / 2$. The regime with small angle limit has been studied in [5] and more recently in $[10,11]$. The question of waveguides with corner arises naturally because it is studied for smooth waveguides in $[13,6,7]$ where we learn, among other things, that curvature induces bound states below the essential spectrum. The idea is that a corner can be seen as an infinite curvature.

The aim of the present paper is to obtain asymptotics for three dimensional cones in the small aperture limit. As in two dimensions, this question naturally appears when looking for the ground states in the small aperture regime of the conical layer studied in [14].

* IRMAR, Univ. Rennes 1, CNRS, Campus de Beaulieu, F-35042 Rennes cedex, France thomas.ourmieres@univ-rennes.1.fr 
We can apply our result to obtain asymptotics for geometrical domains close enough to cones, as spherical sectors, for instance. It yields results in the spirit of [17] in a higher dimension: it is the three dimensional equivalent of the circular sector, the Bessel functions playing a similar role as trigonometric functions.

\subsection{The Dirichlet Laplacian on conical families}

Let us denote by $\left(x_{1}, x_{2}, x_{3}\right)$ the Cartesian coordinates of the space $\mathbb{R}^{3}$ and by $\mathbf{0}=(0,0,0)$ the origin. The positive Laplace operator is given by $-\partial_{1}^{2}-\partial_{2}^{2}-\partial_{3}^{2}$. We are interested in domains delimited by a finite cone. For $\theta \in\left(0, \frac{\pi}{2}\right]$, we introduce the filled cone $\operatorname{Co}(\theta)$ defined by:

$$
\operatorname{Co}(\theta):=\left\{\left(x_{1}, x_{2}, x_{3}\right) \in \mathbb{R}^{3}:-1<x_{3}<0 \quad \text { and }\left(x_{1}^{2}+x_{2}^{2}\right) \cot ^{2} \theta<\left(x_{3}+1\right)^{2}\right\} .
$$

The angle $\theta$ represents the half opening angle of the filled cone. The aim of this paper is the investigation of the lowest eigenvalues of each fibers of the positive Dirichlet Laplacian $-\Delta_{\mathrm{Co}(\theta)}^{\mathrm{Dir}}$ in the small aperture limit.

Remark $1 \operatorname{Co}(\theta)$ being a convex domain, we know that $\operatorname{Dom}\left(-\Delta_{\operatorname{Co}(\theta)}^{\operatorname{Dir}}\right)=H^{2}(\operatorname{Co}(\theta)) \cap H_{0}^{1}(\operatorname{Co}(\theta))$.

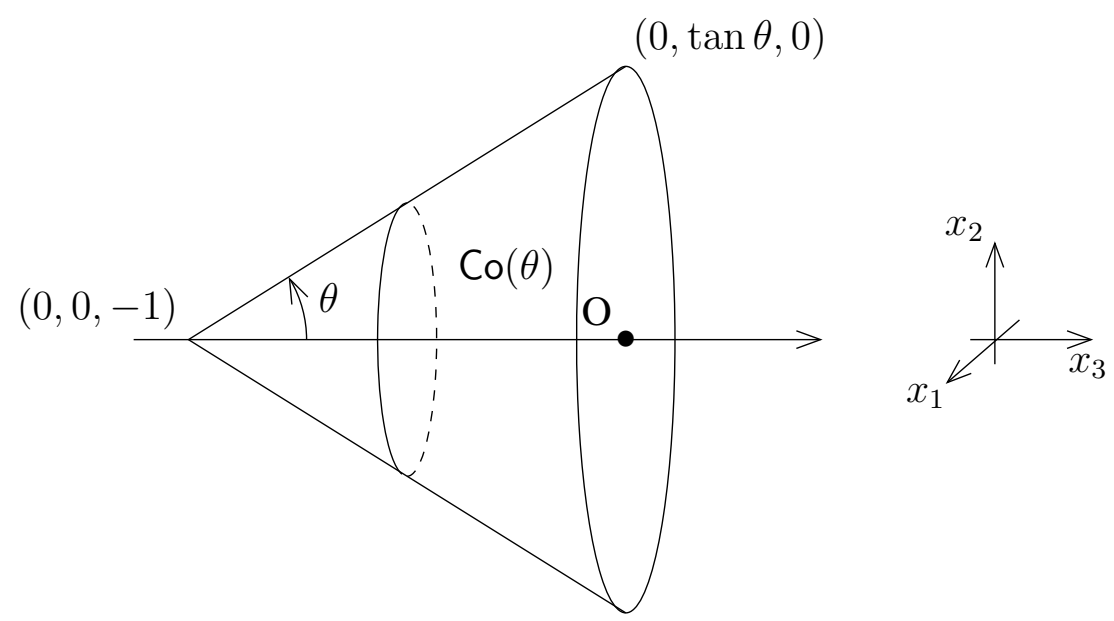

Figure 1: The cone $\operatorname{Co}(\theta)$

\subsection{Structure of the paper}

One can show that after the use of adapted coordinates and a Fourier transform the Dirichlet Laplacian on the cone $\operatorname{Co}(\theta)$ reduces to a countable family of two dimensional semi-classical operators. This is discussed in Section 2.

In Section 3 we state the main theorem and we apply it to a spherical sector. We also go about the so called Born-Oppenheimer approximation. Numerical experiments motivate and illustrate the study.

Afterwards, in Section 4, we perform a change of variables that transforms the meridian triangle into a rectangle. The operator is more complicated but we deal with a simpler geometrical domain. Thanks to this substitution we can construct quasimodes for each operator of the countable family using 
some lemmas which are adapted from the Fredholm alternative. The proof of Theorem 3 about the asymptotics of the first eigenvalues of the cone is over, when, using a Feshbach-Grushin projection, we justify that the Born-Oppenheimer approximation is actually an approximation of our problem and we obtain the separation of the eigenvalues.

We conclude by Section A illustrating by numerical experiments the shape of the eigenfunctions which illustrates some theoretical results obtained all along the paper.

\section{Fiber decomposition}

In this section, we describe the fiber decomposition of the Dirichlet Laplacian on the cone $\operatorname{Co}(\theta)$. We use the terminology detailed in [23, Section XIII.16].

\subsection{Partial wave decomposition}

We are interested in the positive Laplace operator on the cone $\operatorname{Co}(\theta)$ which writes

$$
-\Delta_{\mathrm{Co}(\theta)}^{\mathrm{Dir}}=-\partial_{1}^{2}-\partial_{2}^{2}-\partial_{3}^{2}
$$

We can describe the domain $\operatorname{Co}(\theta)$ using cylindrical coordinates. Let us perform the change of variables and introduce $(r, \phi, z)$ such that

$$
r=\sqrt{x_{1}^{2}+x_{2}^{2}}, \quad \phi=\arctan \frac{x_{2}}{x_{1}}, \quad z=x_{3} .
$$

The cartesian domain $\operatorname{Co}(\theta)$ is transformed into $\operatorname{Tri}(\theta) \times \mathbb{S}^{1}$ where the meridian domain $\operatorname{Tr}(\theta)$ is:

$$
\operatorname{Tri}(\theta):=\left\{(r, z) \in \mathbb{R}^{2}:-1<z<0 \quad \text { and } 0<r<(z+1) \tan \theta\right\} .
$$
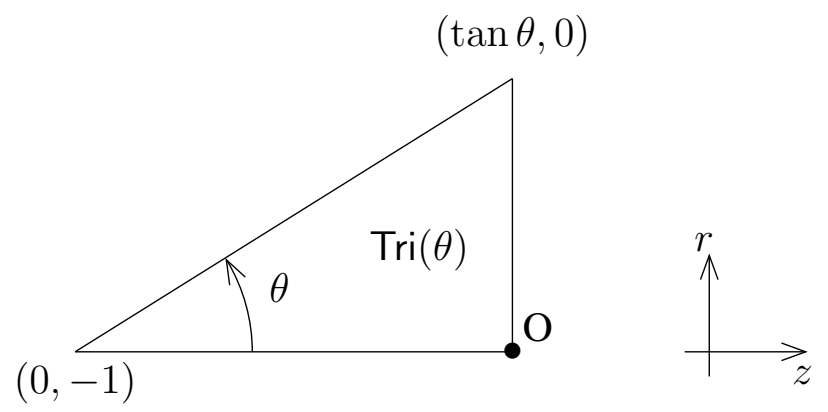

Figure 2: Meridian domain $\operatorname{Tri}(\theta)$

Performing the change of variables the Dirichlet Laplacian is written, on the geometrical domain $\operatorname{Tri}(\theta) \times \mathbb{S}^{1}$, as the operator :

$$
\mathcal{H}_{\operatorname{Tri}(\theta) \times \mathbb{S}^{1}}:=-\frac{1}{r} \partial_{r}\left(r \partial_{r}\right)-\frac{1}{r^{2}} \partial_{\phi}^{2}-\partial_{z}^{2},
$$

its domain being deduced by the change of variables (1). 
The domain $\operatorname{Tri}(\theta) \times \mathbb{S}^{1}$ being axisymmetric we perform a Fourier transform and we have the constant fiber direct sum:

$$
L^{2}\left(\operatorname{Tri}(\theta) \times \mathbb{S}^{1}, r \mathrm{~d} r \mathrm{~d} \phi \mathrm{d} z\right)=L^{2}(\operatorname{Tri}(\theta), r \mathrm{~d} r \mathrm{~d} z) \otimes L^{2}\left(\mathbb{S}^{1}\right)=\bigoplus_{m \in \mathbb{Z}} L^{2}(\operatorname{Tri}(\theta), r \mathrm{~d} r \mathrm{~d} z)
$$

where $L^{2}\left(\mathbb{S}^{1}\right)$ refers to functions on the unit circle with the orthonormal basis $\left\{e^{2 i \pi m \phi}: m \in \mathbb{Z}\right\}$. The operator $\mathcal{H}_{\operatorname{Tr}(\theta) \times \mathbb{S}^{1}}$ decomposes as

$$
\mathcal{H}_{\operatorname{Tr}(\theta) \times \mathbb{S}^{1}}=\bigoplus_{m \in \mathbb{Z}} \mathcal{H}_{\operatorname{Tri}(\theta)}^{[m]}, \quad \text { with } \mathcal{H}_{\operatorname{Tr}(\theta)}^{[m]}=-\frac{1}{r} \partial_{r}\left(r \partial_{r}\right)-\partial_{z}^{2}+\frac{m^{2}}{r^{2}}
$$

where the $\mathcal{H}_{\mathrm{Tri}(\theta)}^{[m]}$ are the fibers of $\mathcal{H}_{\operatorname{Tri}(\theta) \times \mathbb{S}^{1}}$ and their domains $\operatorname{Dom}\left(\mathcal{H}_{\mathrm{Tri}(\theta)}^{[m]}\right)$ are implicitly defined by the decomposition. The fibers $\mathcal{H}_{\text {Tri }(\theta)}^{[m]}$ have compact resolvent, consequently their spectra $\mathfrak{S}\left(\mathcal{H}_{\text {Tri }(\theta)}^{[m]}\right)$ consist in non-decreasing sequences of eigenvalues. We have :

$$
\mathfrak{S}\left(\mathcal{H}_{\operatorname{Tri}(\theta) \times \mathbb{S}^{1}}\right)=\cup_{m \in \mathbb{Z}} \mathfrak{S}\left(\mathcal{H}_{\operatorname{Tri}(\theta)}^{[m]}\right)
$$

Thus, if we denote by $\mu_{n}^{[m]}(\theta)$ the $n^{\text {th }}$ eigenvalue of $\mathcal{H}_{\mathrm{Tri}(\theta)}^{[m]}$ we have the following description of the sprectum:

$$
\mathfrak{S}\left(\mathcal{H}_{\operatorname{Tri}(\theta) \times \mathbb{S}^{1}}\right)=\underset{(n, m) \in \mathbb{N}^{*} \times \mathbb{Z}}{\cup}\left\{\mu_{n}^{[m]}(\theta)\right\}
$$

Remark 2 For $\psi \in \operatorname{Dom}\left(\mathcal{H}_{\operatorname{Tr}(\theta)}^{[m]}\right)$, we have the Dirichlet boundary condition $\psi(r, 0)=0$ and $\psi((z+1) \tan \theta, z)=0$.

If $m \neq 0$, we have for integrability reasons $\psi(0, z)=0$. We refer to [4, Chapt. II] for more information.

\subsection{Rescaling of the meridian domain $\operatorname{Tr}(\theta)$}

We rescale the integration domain in order to avoid its dependence on $\theta$. Therefore, this dependence is transferred in the coefficients of the operator. For this reason, let us perform the following linear change of coordinates:

$$
x=z, \quad y=\frac{1}{\tan \theta} r
$$

which maps $\operatorname{Tri}(\theta)$ onto $\operatorname{Tri}\left(\frac{\pi}{4}\right)$. That is why we set for simplicity:

$$
\operatorname{Tri}:=\operatorname{Tri}\left(\frac{\pi}{4}\right)
$$

Then, for each $m \in \mathbb{Z}, \mathcal{H}_{\mathrm{Tri}(\theta)}^{[m]}$ is unitary equivalent to the operator with the new integration domain Tri:

$$
\mathcal{D}^{[m]}(\theta):=-\partial_{x}^{2}-\frac{1}{y \tan ^{2} \theta} \partial_{y}\left(y \partial_{y}\right)+\frac{m^{2}}{y^{2} \tan ^{2} \theta} .
$$

with implicit boundary conditions as in Remark 2 . We let $h=\tan \theta$; after a multiplication by $\tan ^{2} \theta$, we get the new operator: 


$$
\mathcal{L}^{[m]}(h):=-h^{2} \partial_{x}^{2}-\frac{1}{y} \partial_{y}\left(y \partial_{y}\right)+\frac{m^{2}}{y^{2}} .
$$

This operator is partially semi-classical in $x$. It is the shape of $\mathcal{L}^{[m]}(h)$ that leads us in each steps of our study. That is why, in Subsection 3.3, we first consider its Born-Oppenheimer approximation (see $[8,20,22])$. Then our reasoning is inspired by the philosophy presented in $[16,19,18]$.

\section{Numerical motivations and main results}

\subsection{Asymptotic expansion of eigenvalues}

According to the structure of the spectrum established in (3), we recall that we denote by $\mu_{n}^{[m]}(\theta)$ the $n^{\text {th }}$ eigenvalue of the $m^{\text {th }}$ fiber of $\mathcal{H}_{\mathrm{Co}(\theta)}$. In order to get more detailed information about the behavior of $\mu_{n}^{[m]}(\theta)$ we can, at first, study it numerically. We carried out the computation with the operator $\mathcal{L}^{[m]}(\tan \theta)$ defined in (6) and we pictured its eigenvalues denoted $\lambda_{n}^{[m]}(\tan \theta)$.

Figures 3 suggests that, for $m=0,1,2$, the eigenvalues converge to a certain limit as the aperture $\theta$ goes to 0 . Moreover, this value is near $j_{m, 1}^{2}$, where we denote by $j_{m, 1}$ the first zero of the $m^{\text {th }}$ Bessel function of first kind, represented by black dots. This result has to be connected to the one established in [14] where, studying a conical layer, the value $\frac{j_{0,1}^{2}}{\pi^{2}}$ plays a similar role. In that paper, they only consider the operator from the fiber of order 0 because in this case the other fibers have only essential spectrum (the factor $\frac{1}{\pi^{2}}$ being a normalization constant). One can see that for $\theta$ large enough the eigenvalues cross and although $\lambda_{n}^{[m]}$ represents the $n^{\text {th }}$ eigenvalue of the $m^{\text {th }}$ fiber of $\mathcal{L}^{[m]}(\tan \theta)$ it is clear that $\lambda_{n}^{[0]}(\tan \theta)$ is not necessarily its $n^{\text {th }}$ eigenvalue.

The main result of this paper is not only the convergence of the eigenvalues as $\theta \rightarrow 0$ illustrated in Figure 3 but an asymptotic expansion of these eigenvalues. Indeed, the lowest eigenvalues of each fiber of $\mathcal{H}_{\operatorname{Co}(\theta)}$ admit expansions at any order in powers of $\theta^{1 / 3}$. We first state the result for the scaled operators $\mathcal{L}^{[m]}(h)$ introduced in (6):

Theorem 3 If we denote by $z_{\mathrm{A}}(n)$ the $n^{\text {th }}$ zero of the reversed Airy function, the eigenvalues of $\mathcal{L}^{[m]}(h)$, denoted by $\lambda_{n}^{[m]}(h)$, admit the expansions:

$$
\lambda_{n}^{[m]}(h) \underset{h \rightarrow 0}{\sim} \sum_{k \geq 0} \beta_{k, n}^{[m]} h^{k / 3} \quad \text { with } \beta_{0, n}^{[m]}=j_{m, 1}^{2}, \beta_{1, n}^{[m]}=0, \beta_{2, n}^{[m]}=\left(2 j_{m, 1}^{2}\right)^{2 / 3} z_{\mathrm{A}}(n),
$$

the terms of odd rank being zero for $j \leq 8$. The corresponding eigenfunctions have expansions in powers of $h^{1 / 3}$ with both scales $x / h^{2 / 3}$ and $x / h$.

In terms of the physical domain $\operatorname{Tri}(\theta)$, we immediately deduce from the previous theorem that the eigenvalues of the $m^{- \text {th }}$ fiber of $-\Delta_{\operatorname{Co}(\theta)}^{\operatorname{Dir}}$ admit the expansions:

$$
\mu_{n}^{[m]}(\theta) \underset{\theta \rightarrow 0}{\sim} \frac{1}{\theta^{2}} \sum_{k \geq 0} \beta_{k, n}^{[m], \Delta} \theta^{k / 3} \quad \text { with } \beta_{0, n}^{[m], \Delta}=j_{m, 1}^{2}, \beta_{1, n}^{[m], \Delta}=0, \beta_{2, n}^{[m], \Delta}=\left(2 j_{m, 1}\right)^{2 / 3} z_{\mathrm{A}}(n) .
$$

Figure 4 depicts that for small aperture $\theta$, the numerical eigenvalues $\lambda_{n}^{[0]}(\tan \theta)$ match with the theoretical expected behavior deduced from Theorem 3. The stalling for very small values of the aperture is due to numerical difficulties when $\theta \rightarrow 0$. 


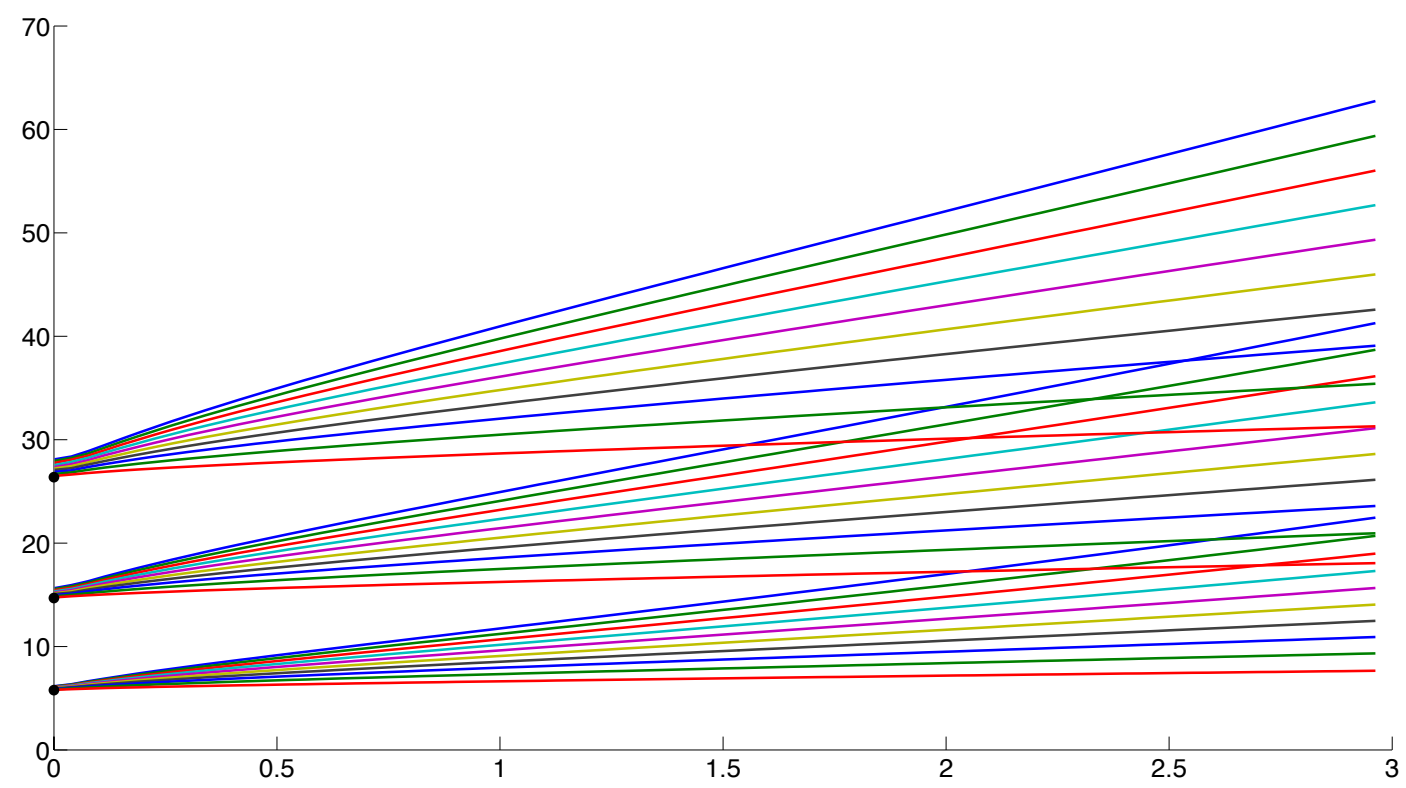

Figure 3: This figure represents the dependence of the first ten eigenvalues $\lambda_{n}^{[m]}(\tan \theta)(m=0,1,2)$ on the aperture $\theta$ (in degrees). We computed each eigenvalue for 80 values of $\theta$.

\subsection{Application to the spherical cone}

Theorem 3 on the cone $\operatorname{Co}(\theta)$ is closely related to the Dirichlet problem on a spherical cone. We denote by $\operatorname{Sph}(\theta)$ the spherical cone of radius 1 and aperture $\theta$ with center in $(0,0,-1)$ described in Figure 5. We have

$$
-\Delta_{\mathrm{Sph}(\theta)}^{\mathrm{Dir}}:=-\partial_{1}^{2}-\partial_{2}^{2}-\partial_{3}^{2}
$$

We perform the change of variables

$\rho=\sqrt{x_{1}^{2}+x_{2}^{2}+\left(x_{3}+1\right)^{2}}, \quad \alpha=\operatorname{arcos}\left(\frac{x_{3}+1}{\rho}\right), \quad \beta=\left\{\begin{array}{cc}\operatorname{arcos}\left(\frac{x_{1}}{\sqrt{x_{1}^{2}+x_{2}^{2}}}\right) & \text { if } \quad x_{2} \geq 0, \\ 2 \pi-\operatorname{arcos}\left(\frac{x_{1}}{\sqrt{x_{1}^{2}+x_{2}^{2}}}\right) & \text { if } \quad x_{2}<0 .\end{array}\right.$

Hence the domain $\operatorname{Sph}(\theta)$ is transformed into

$$
\widehat{\operatorname{Sph}}(\theta)=\widehat{\operatorname{Circ}(\theta)} \times \mathbb{S}^{1},
$$

where $\widehat{\operatorname{Circ}(\theta)}$ is the circular meridian domain in the coordinates $(\rho, \alpha)$.

Remark 4 If instead of the change of variables (7) we change into cylindrical coordinates as in (1), the associated meridian circular sector $\operatorname{Circ}(\theta)$ is the one of Figure 6. One can pass from $\widehat{\operatorname{Circ}}(\theta)$ to $\operatorname{Circ}(\theta)$ by the change of variables

$$
r=\rho \cos \alpha-1, \quad z=\rho \sin \alpha,
$$

which links those two domains without the cartesian domain. 


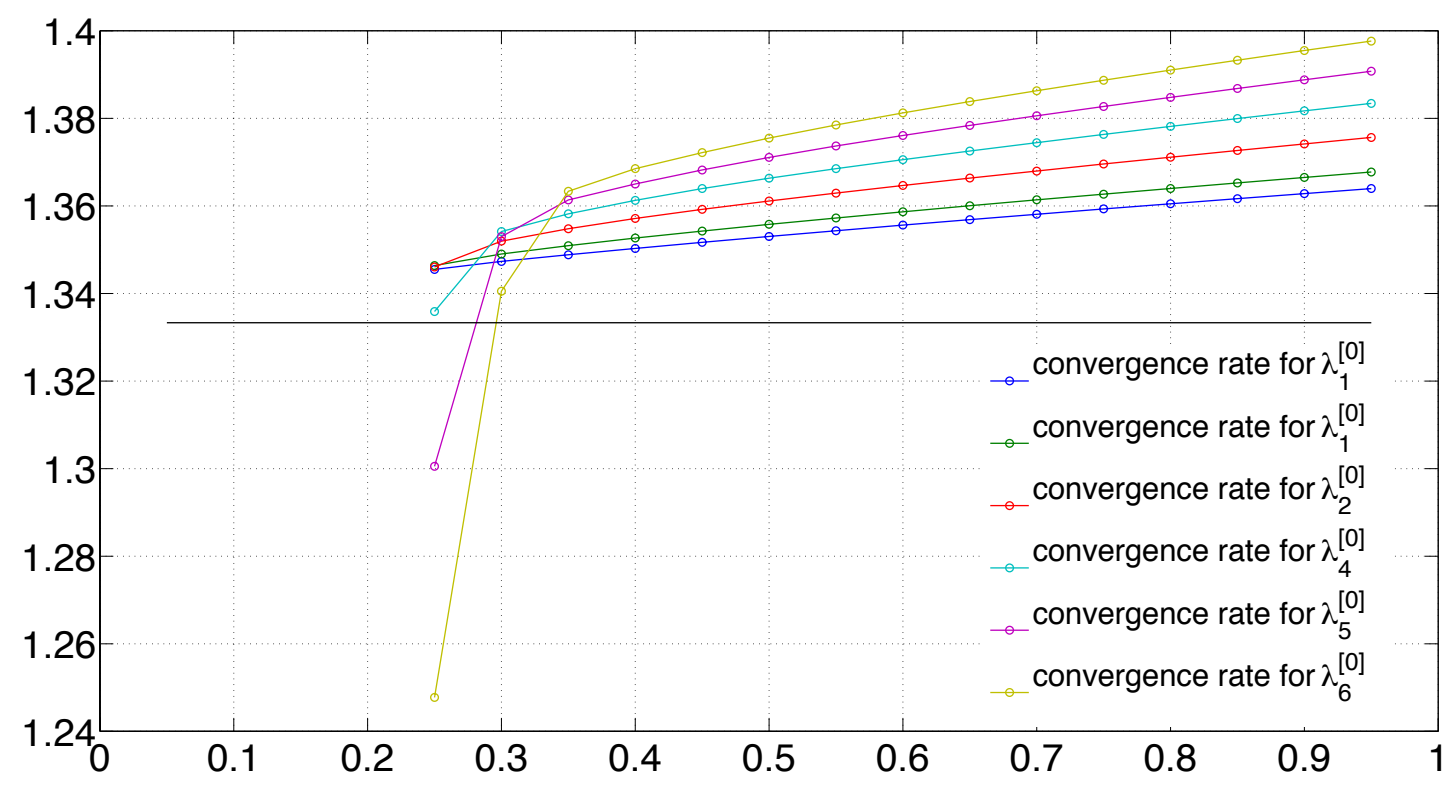

Figure 4: This figure represents the convergence rate of the first six eigenvalues $\lambda_{n}^{[0]}(\tan \theta)$ to the two first terms of the theoretical asymptotics on the aperture $\theta$ (in degrees). The black line represents the value $4 / 3$.

The Dirichlet Laplacian $\mathcal{H}_{\widehat{\mathrm{Sph}}(\theta)}$ writes in spherical coordinates

$$
\mathcal{H}_{\widehat{\operatorname{Sph}}(\theta)}:=-\frac{1}{\rho^{2}} \partial_{\rho}\left(\rho^{2} \partial_{\rho}\right)-\frac{1}{\rho^{2} \sin \alpha} \partial_{\alpha}\left(\sin \alpha \partial_{\alpha}\right)-\frac{1}{\rho^{2} \sin ^{2} \alpha} \partial_{\beta}^{2},
$$

on $L^{2}\left(\widehat{\operatorname{Sph}}(\theta), \rho^{2} \sin \alpha \mathrm{d} \rho \mathrm{d} \alpha \mathrm{d} \beta\right)$. As in Section 2 we have the constant fiber direct sum:

$$
L^{2}\left(\widehat{\operatorname{Sph}}(\theta), \rho^{2} \sin \alpha \mathrm{d} \rho \mathrm{d} \alpha \mathrm{d} \beta\right)=\bigoplus_{m \in \mathbb{Z}} L^{2}\left(\widehat{\operatorname{Circ}(\theta)}, \rho^{2} \sin \alpha \mathrm{d} \rho \mathrm{d} \alpha\right),
$$

and $\mathcal{H}_{\widehat{\mathrm{Sph}}(\theta)}$ decomposes in fibers :

$$
\mathcal{H}_{\widehat{\operatorname{Sph}}(\theta)}=\bigoplus_{m \in \mathbb{Z}} \mathcal{H}_{\widehat{\operatorname{Circ}}(\theta)}^{[m]}
$$

where

$$
\mathcal{H}_{\frac{\operatorname{Circ}(\theta)}{[m]}}:=-\frac{1}{\rho^{2}} \partial_{\rho}\left(\rho^{2} \partial_{\rho}\right)-\frac{1}{\rho^{2} \sin \alpha} \partial_{\alpha}\left(\sin \alpha \partial_{\alpha}\right)+\frac{m^{2}}{\rho^{2} \sin ^{2} \alpha},
$$

with implicit domains and boundary conditions. Let $(\mu, \Psi)$ be an eigenpair of $\mathcal{H}_{\overline{\operatorname{Circ}}(\theta)}^{[m]}$, with $\Psi(\rho, \alpha)=R(\rho) M(\alpha)$. It should satisfy the following system of differential equations :

$$
\left\{\begin{array}{c}
{\left[\partial_{\rho}\left(\rho^{2} \partial_{\rho}\right)+\left(c(\theta)-\mu \rho^{2}\right)\right] R(\rho)=0} \\
{\left[-\frac{1}{\sin \alpha} \partial_{\alpha}\left(\sin \alpha \partial_{\alpha}\right)+\frac{m^{2}}{\sin ^{2} \alpha}\right] M(\alpha)=c(\theta) M(\alpha) .}
\end{array}\right.
$$


Remark 5 We are not interested here in solving those equations. Nevertheless one can see that formally, when $\theta \rightarrow 0$ the angle $\alpha$ is small and the last equation of (8) looks like the Bessel equation. This could be a lead to find an asymptotic expansion at any order of $\mu$ when $\theta \rightarrow 0$.

However, thanks to Theorem 3 , we have easily a finite term asymptotic for the eigenvalues of $-\Delta_{\mathrm{Sph}(\theta)}^{\mathrm{Dir}}$. Let $\operatorname{Co}(\theta, \cos \theta)$ be the set $\operatorname{Co}(\theta)$ up to a dilatation of ratio $\cos \theta$. We have the set inclusion in $\mathbb{R}^{3}$

$$
\operatorname{Co}(\theta, \cos \theta) \subset \operatorname{Sph}(\theta) \subset \operatorname{Co}(\theta) .
$$

Let $\breve{\mu}_{n}(\theta)$ be the $n^{\text {th }}$ eigenvalue of the Dirichlet Laplacian on $\operatorname{Sph}(\theta)$ and $\mu_{n}(\theta)$ the one on the cone $\operatorname{Co}(\theta)$, the monotonicity of the Dirichlet Laplacian yields:

$$
\left(1+\tan ^{2} \theta\right) \mu_{n}(\theta) \geq \breve{\mu}_{n}(\theta) \geq \mu_{n}(\theta)
$$

If $\breve{\mu}_{n}^{[m]}(\theta)$ denotes the $n^{\text {th }}$ eigenvalue of the $m^{\text {th }}$ fiber of the Dirichlet Laplacian, (9) yields for small $\theta$

$$
\left(1+\tan ^{2} \theta\right) \mu_{n}^{[0]}(\theta) \geq \breve{\mu}_{n}^{[0]}(\theta) \geq \mu_{n}^{[0]}(\theta) .
$$

To deal with higher fibers we can apply the exact same argument on the meridian circular sector $\operatorname{Cir}(\theta)$ and the meridian triangle $\operatorname{Tri}(\theta)$ because :

$$
\operatorname{Tri}(\theta, \cos \theta) \subset \operatorname{Cir}(\theta) \subset \operatorname{Tri}(\theta) .
$$

As for $m \geq 1$, there is a Dirichlet boundary condition everywhere (see Remark 2) we have :

$$
\left(1+\tan ^{2} \theta\right) \mu_{n}^{[m]}(\theta) \geq \breve{\mu}_{n}^{[m]}(\theta) \geq \mu_{n}^{[m]}(\theta) .
$$

Those inequalities provide the first terms in the asymptotics of $\breve{\mu}_{n}^{[m]}(\theta)$.

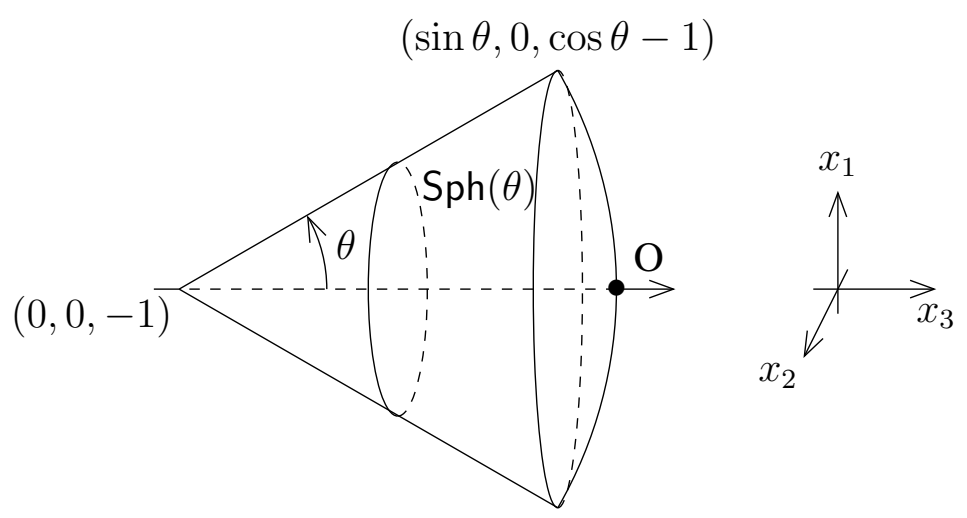

Figure 5: The spherical cone $\operatorname{Sph}(\theta)$

\subsection{Schrödinger operators in one dimension}

In the analysis of $\mathcal{L}^{[m]}(h)$ we will see that its so called Born-Oppenheimer approximation will play an important role. That is why we define

$$
l_{\mathrm{BO}}^{[m]}(h):=-h^{2} \partial_{x}^{2}+v^{[m]}(x),
$$



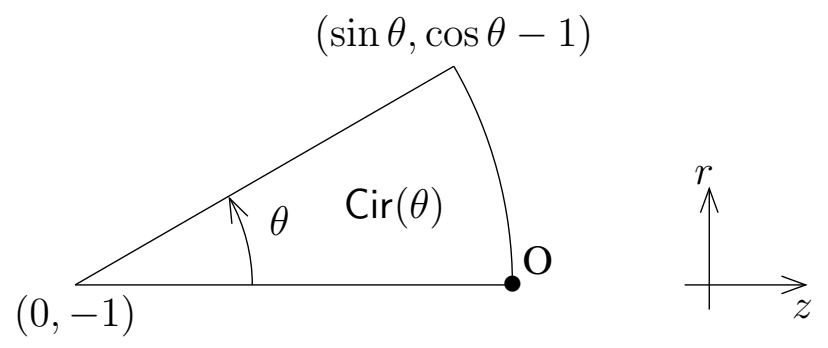

Figure 6: Meridian domain $\operatorname{Cir}(\theta)$

where the effective potential $v^{[m]}$ is obtained by replacing $-\frac{1}{y} \partial_{y}\left(y \partial_{y}\right)+\frac{m^{2}}{y^{2}}$ in the expression of $\mathcal{L}^{[m]}(h)$ by its lowest eigenvalue on each slice of Tri at fixed $x$. One can see that

$$
v^{[m]}(x)=\frac{j_{m, 1}^{2}}{(x+1)^{2}} \quad \text { for } x \in(-1,0)
$$

By construction, for any $m$, the operator (10) can be seen as a lower bound of the operator $\mathcal{L}^{[m]}(h)$. As it is shown in subsection 4.4, the choice of this approximation gives us informations about the eigenvalues of $\mathcal{H}_{\operatorname{Tr}(\theta) \times \mathbb{S}^{1}}$. We have the

Proposition 6 The eigenvalues of $l_{\mathrm{BO}}^{[m]}(h)$, denoted by $\lambda_{\mathrm{BO}, n}^{[m]}(h)$, admit the expansion:

$$
\lambda_{\mathrm{BO}, n}^{[m]}(h) \underset{h \rightarrow 0}{\sim} \sum_{k \geq 0} \hat{\beta}_{k, n}^{[m]} h^{2 j / 3}, \quad \text { with } \hat{\beta}_{0, n}^{[m]}=j_{m, 1}^{2} \text { and } \hat{\beta}_{1, n}^{[m]}=\left(2 j_{m, 1}^{2}\right)^{2 / 3} z_{\mathrm{A}}(n) .
$$

The shape of the effective potential $v^{[m]}$ is the same as in [11, Section 3]. Then, we deduce Proposition 6 from [11, Th 4.1.]. The key is the construction of quasimodes at the scale $h^{2 / 3}$ which naturally arises by expanding the effective potential $v^{[m]}$ and recognizing the Airy operator at first order. This method is adapted from the harmonic approximation for regular potentials with a well (see [24], [9, Chapt. 11] and [12, Chapt. 4]). It yields an upper bound of the eigenvalues of $l_{\mathrm{BO}}^{[m]}(h)$. To obtain a lower bound we then need the Agmon estimates of Propositions 7 and 8 and to apply the min-max principle and get the separation of eigenvalues.

The Agmon estimates near $x=0$ take the following form

Proposition 7 Let $\Gamma_{0}>0$. There exist $h_{0}>0, C_{0}>0$ and $\eta_{0}>0$ such that for any $h \in\left(0, h_{0}\right)$ and all eigenpair $(\lambda, \psi)$ of $l_{\mathrm{BO}}^{[m]}(h)$ satisfying $\left|\lambda-j_{m, 1}^{2}\right| \leq \Gamma_{0} h^{2 / 3}$, we have:

$$
\int_{-1}^{0} e^{\eta_{0} h^{-1}|x|^{3 / 2}}\left(|\psi|^{2}+\left|h^{2 / 3} \partial_{x} \psi\right|^{2}\right) \mathrm{d} x \leq C_{0}\|\psi\|^{2}
$$

And the Agmon estimates near $x=-1$ take the following form

Proposition 8 Let $\Gamma_{0}>0$ and $\rho_{0} \in\left(0, j_{m, 1}\right)$. There exist $h_{0}>0, C_{0}>0$ such that for any $h \in\left(0, h_{0}\right)$ and all eigenpair $(\lambda, \psi)$ of $l_{\mathrm{BO}}^{[m]}(h)$ satisfying $\left|\lambda-j_{m, 1}^{2}\right| \leq \Gamma_{0} h^{2 / 3}$, we have:

$$
\int_{-1}^{0}(x+1)^{-\rho_{0} / h}\left(|\psi|^{2}+\left|h \partial_{x} \psi\right|^{2}\right) \mathrm{d} x \leq C_{0}\|\psi\|^{2} .
$$


These semiclassical Agmon estimates (see [1, 2]) are obtained using the technical background of [12, Chapt. 6] and [19].

\section{Meridian triangle Tri with Dirichlet boundary condition}

The aim of this section is to prove Theorem 3. The proof will be divided into two main steps : a construction of quasimodes and the use of the true eigenfunctions of $\mathcal{L}^{[m]}(h)$ as quasimodes for the Born-Oppenheimer approximation in order to obtain a lower bound for true eigenvalues. We first perform a change of variables to transform the triangle into the square:

$$
u=x \in(-1,0), \quad t=\frac{y}{x+1} \in(0,1)
$$

The meridian triangle Tri is transformed into a square $\mathrm{Sq}$

$$
\mathrm{Sq}:=(-1,0) \times(0,1) \text {. }
$$

The operator $\mathcal{L}^{[m]}(h)$ becomes:

$$
\begin{aligned}
\mathcal{L}_{\mathrm{Sq}}^{[m]}(h)\left(u, t ; \partial_{u}, \partial_{t}\right):= & \frac{1}{(u+1)^{2}}\left(-\frac{1}{t} \partial_{t}\left(t \partial_{t}\right)+\frac{m^{2}}{t^{2}}\right)-h^{2} \partial_{u}^{2} \\
& -\frac{h^{2} t^{2}}{(u+1)^{2}} \partial_{t}^{2}+\frac{2 h^{2} t}{u+1} \partial_{t} \partial_{u}-\frac{2 h^{2} t}{(u+1)^{2}} \partial_{t},
\end{aligned}
$$

on $L^{2}\left(\mathrm{Sq}, t(u+1)^{2} \mathrm{~d} u \mathrm{~d} t\right)$ with Dirichlet boundary condition on the faces $\{(0, t): 0<t<1\}$ and $\{(u, 1):-1<u<0\}$. The equation $\mathcal{L}^{[m]}(h) \psi_{h}^{[m]}=\lambda_{h}^{[m]} \psi_{h}^{[m]}$ is transformed into the equation

$$
\mathcal{L}_{\mathrm{Sq}}^{[m]}(h) \hat{\psi}_{h}^{[m]}=\lambda_{h}^{[m]} \hat{\psi}_{h}^{[m]} \quad \text { with } \quad \hat{\psi}_{h}^{[m]}(u, t)=\psi_{h}^{[m]}(x, y) .
$$

In what follows we denote by $\langle\cdot, \cdot\rangle_{t}$ the scalar product on $L^{2}((0,1), t \mathrm{~d} t)$.

\subsection{Quasimodes}

This section is devoted to the proof of the following proposition.

Proposition 9 If $\mathfrak{S}\left(\mathcal{L}^{[m]}(h)\right)$ denotes the spectrum of $\mathcal{L}^{[m]}(h)$, there are sequences $\left(\beta_{j, n}^{[m]}\right)_{j \geq 0}$ for any integer $n \geq 1$ so that there holds: for all $N_{0} \in \mathbb{N}^{*}$ and $J \in \mathbb{N}$, there exist $h_{0}>0$ and $C>0$ such that for $h \in\left(0, h_{0}\right)$

$$
\operatorname{dist}\left(\mathfrak{S}\left(\mathcal{L}^{[m]}(h)\right), \sum_{j=0}^{J} \beta_{j, n}^{[m]} h^{j / 3}\right) \leq C h^{(J+1) / 3}, \quad n=1, \ldots, N_{0}
$$

Moreover, we have: $\beta_{0, n}^{[m]}=j_{m, 1}^{2}, \beta_{1, n}^{[m]}=0$, and $\beta_{2, n}^{[m]}=\left(2 j_{m, 1}^{2}\right)^{2 / 3} z_{\mathrm{A}}(n)$.

Proof: The proof is divided into three parts. The first one deals with the form of the Ansatz chosen to construct quasimodes. The second part deals with three lemmas about operators which appear in the first part. The third part is the determination of the profiles of the Ansatz. 
Shape of the Ansatz We want to construct quasimodes $\left(\beta_{h}^{[m]}, \psi_{h}^{[m]}\right)$ for the operator $\mathcal{L}^{[m]}(h)\left(x, y ; \partial_{x}, \partial_{y}\right)$. It will be more convenient to work on the square Sq with the operator $\mathcal{L}_{\mathrm{Sq}}^{[m]}(h)\left(u, t ; \partial_{u}, \partial_{t}\right)$. We introduce the new scales

$$
s=h^{-2 / 3} u \quad \text { and } \quad \sigma=h^{-1} u,
$$

and we look for quasimodes $\left(\beta_{h}^{[m]}, \hat{\psi}_{h}^{[m]}\right)$ in the form of series

$$
\beta_{h}^{[m]} \sim \sum_{j \geq 0} \beta_{j}^{[m]} h^{j / 3} \quad \text { and } \quad \hat{\psi}_{h}^{[m]}(u, t) \sim \sum_{j \geq 0}\left(\Psi_{j}^{[m]}(s, t)+\Phi_{j}^{[m]}(\sigma, t)\right) h^{j / 3}
$$

in order to solve $\mathcal{L}_{\mathrm{Sq}}^{[m]}(h) \hat{\psi}_{h}^{[m]}=\beta_{h}^{[m]} \hat{\psi}_{h}^{[m]}$ in the sense of formal series. An Ansatz containing only the scale $h^{-2 / 3}$ is not sufficient to construct quasimodes for $\mathcal{L}_{\mathrm{Sq}}^{[m]}(h)$ because one can see that the system is overdetermined. Expanding the operator in powers of $h^{2 / 3}$ we obtain the formal series:

$$
\mathcal{L}_{\mathrm{Sq}}^{[m]}(h)\left(h^{2 / 3} s, t ; h^{-2 / 3} \partial_{s}, \partial_{t}\right) \sim \sum_{j \geq 0} \mathcal{L}_{2 j}^{[m]} h^{2 j / 3} \quad \text { with leading term } \quad \mathcal{L}_{0}^{[m]}=-\frac{1}{t} \partial_{t}\left(t \partial_{t}\right)+\frac{m^{2}}{t^{2}},
$$

and in power of $h$ :

$$
\mathcal{L}_{\mathrm{Sq}}^{[m]}(h)\left(h \sigma, t ; h^{-1} \partial_{\sigma}, \partial_{t}\right) \sim \sum_{j \geq 0} \mathcal{N}_{3 j}^{[m]} h^{j} \quad \text { with leading term } \quad \mathcal{N}_{0}^{[m]}=\left(-\frac{1}{t} \partial_{t}\left(t \partial_{t}\right)+\frac{m^{2}}{t^{2}}\right)-\partial_{\sigma}^{2}
$$

In what follows, in order to ensure the Dirichlet condition on Tri $\backslash(-1,0) \times\{0\}$ we will require for our Ansatz the boundary condition, for any $j \in \mathbb{N}$ :

$$
\begin{gathered}
\Psi_{j}^{[m]}(0, t)+\Phi_{j}^{[m]}(0, t)=0, \quad 0 \leq t \leq 1, \\
\Psi_{j}^{[m]}(s, 1)=0, \quad s<0 \quad \text { and } \quad \Phi_{j}^{[m]}(\sigma, 1)=0, \quad \sigma \leq 0 .
\end{gathered}
$$

More specifically, we are interested in the ground energy $\lambda=j_{m, 1}^{2}$ of the Dirichlet problem at 1 for $\mathcal{L}_{0}^{[m]}$ on the interval $(0,1)$. Thus, we have to solve the Dirichlet problem for the operators $\mathcal{N}_{0}^{[m]}-j_{m, 1}^{2}$ and $\mathcal{L}_{0}^{[m]}-j_{m, 1}^{2}$ on the half-strip

$$
\text { Hst }=\mathbb{R}_{-} \times(0,1),
$$

and look for solutions exponentially decaying (see Remark 10). Our aim is to apply the spectral theorem to the truncated series $\hat{\psi}_{h}^{[m]}(u, t)$ restricted in the square Sq thanks to a cut-off function.

Remark 10 In the following part, we will need to use exponentially decaying functions. Then, we define the spaces:

$$
\begin{gathered}
L_{\exp }^{2}\left(\mathbb{R}_{-}\right):=\left\{f \in L^{2}\left(\mathbb{R}_{-}\right): \exists \alpha>0 \text { such that } e^{\alpha|s|} f \in L^{2}\left(\mathbb{R}_{-}\right)\right\}, \\
H_{\text {exp }}^{2}\left(\mathbb{R}_{-}\right):=\left\{f \in H^{2}\left(\mathbb{R}_{-}\right): \exists \alpha>0 \text { such that } e^{\alpha|s|} f \in H^{2}\left(\mathbb{R}_{-}\right)\right\}, \\
L_{\exp }^{2}(\mathrm{Hst}, t \mathrm{~d} u \mathrm{~d} t):=\left\{f \in L^{2}(\mathrm{Hst}, t \mathrm{~d} u \mathrm{~d} t): \exists \alpha>0 \text { such that } e^{\alpha|u|} f \in L^{2}(\mathrm{Hst}, t \mathrm{~d} u \mathrm{~d} t)\right\}, \\
H_{\exp }^{2}(\mathrm{Hst}, t \mathrm{~d} u \mathrm{~d} t):=\left\{f \in H^{2}(\mathrm{Hst}, t \mathrm{~d} u \mathrm{~d} t): \exists \alpha>0 \text { such that } e^{\alpha|u|} f \in H^{2}(\mathrm{Hst}, t \mathrm{~d} u \mathrm{~d} t)\right\} .
\end{gathered}
$$




\section{Three lemmas}

To start the construction of our Ansatz we will need the three next lemmas. Lemmas 12 and 13 are consequences of the Fredholm alternative.

Lemma 11 We denote the $n^{\text {th }}$ normalized eigenfunction of $\mathcal{L}_{0}^{[m]}$ by $b_{n}^{[m]}$ :

$$
b_{n}^{[m]}(t)=C_{n}^{[m]} J_{m}\left(j_{m, n} t\right) \quad\left(C_{n}^{[m]} \in \mathbb{R} \text { being a normalization constant }\right),
$$

where $J_{m}$ is the $m^{\text {th }}$ Bessel function of first kind. Let $F=F(\sigma, t)$ be a function in $L_{\text {exp }}^{2}(\mathrm{Hst}, t \mathrm{~d} \sigma \mathrm{d} t)$ and let $G \in H^{3 / 2}((0,1), t \mathrm{~d} t)$ be a function of $t$ with $G(1)=0$. Then there exists a unique $\gamma \in \mathbb{R}$ such that the problem

$$
\left(\mathcal{N}_{0}^{[m]}-j_{m, 1}^{2}\right) \Phi=F, \quad \Phi(\sigma, 1)=0, \Phi(0, t)=G(t)+\gamma b_{1}^{[m]}(t)
$$

admits a unique solution in $H_{\exp }^{2}(\mathrm{Hst}, t \mathrm{~d} \sigma \mathrm{d} t)$. Moreover $\gamma$ is given by:

$$
\gamma=-\int_{-\infty}^{0} \int_{0}^{1} F(\sigma, t) \sigma b_{1}^{[m]}(t) t \mathrm{~d} t \mathrm{~d} \sigma-\int_{0}^{1} G(t) b_{1}^{[m]}(t) t \mathrm{~d} t
$$

Lemma 12 Let $F=F(s, t)$ be a function in $L_{\exp }^{2}(\mathrm{Hst}, t \mathrm{~d} s \mathrm{~d} t)$. Then, there exists solution $(s) \Psi$ such that:

$$
\left(\mathcal{L}_{0}^{[m]}-j_{m, 1}^{2}\right) \Psi=F \quad \text { in } \quad \mathrm{Hst}, \quad \Psi(s, 1)=0
$$

if and only if $\left\langle F(s, \cdot), b_{1}^{[m]}\right\rangle_{t}=0$ for all $s<0$. In this case, $\Psi(s, t)=\Psi^{\perp}(s, t)+g(s) b_{1}^{[m]}(t)$ where $\Psi^{\perp}$ satisfies $\left\langle\Psi(s, \cdot)^{\perp}, b_{1}^{[m]}\right\rangle_{t} \equiv 0$ and $\Psi$ belongs to $H_{\exp }^{2}(\mathrm{Hst}, t \mathrm{~d} s \mathrm{~d} t)$.

Lemma 13 Let $n \geq 1$. We recall that $z_{\mathrm{A}}(n)$ is the $n^{\text {th }}$ zero of the Airy reverse function, and we denote by

$$
a_{n}^{[m]}(s)=\tilde{C}_{n}^{[m]} \mathrm{A}\left(\left(2 j_{m, 1}^{2}\right)^{1 / 3} s+z_{\mathrm{A}}(n)\right) \quad\left(\tilde{C}_{n}^{[m]} \in \mathbb{R} \text { being a normalization constant }\right)
$$

a normalized eigenfunction of the operator $-\partial_{s}^{2}-\left(2 j_{m, 1}^{2}\right) s$ with Dirichlet condition on $\mathbb{R}_{-}$associated with the eigenvalue $\left(j_{m, 1}^{2}\right)^{2 / 3} z_{\mathrm{A}}(n)$. Let $f=f(s)$ be a function in $L_{\exp }^{2}\left(\mathbb{R}_{-}\right)$and let $c \in \mathbb{R}$. Then there exists a unique $\beta \in \mathbb{R}$ such that the problem:

$$
\left(-\partial_{s}^{2}-2 j_{m, 1}^{2} s-\left(j_{m, 1}^{2}\right)^{2 / 3} z_{\mathrm{A}}(n)\right) g=f+\beta a_{n}^{[m]} \quad \text { in } \mathbb{R}_{-}, \text {with } g(0)=c,
$$

has a unique solution in $H_{\exp }^{2}\left(\mathbb{R}_{-}\right)$.

Remark 14 The key in proving Lemmas 11 and 12 is the decomposition as a tensor product of $L^{2}(\mathrm{Hst}, t \mathrm{~d} \sigma \mathrm{d} t)$ and $L^{2}(\mathrm{Hst}, t \mathrm{~d} s \mathrm{~d} t)$. One can see that $L^{2}(\mathrm{Hst}, t \mathrm{~d} \sigma \mathrm{d} t)=L^{2}\left(\mathbb{R}_{-}, \mathrm{d} \sigma\right) \widehat{\otimes} L^{2}((0,1), t \mathrm{~d} t)$ and $L^{2}(\mathrm{Hst}, t \mathrm{~d} s \mathrm{~d} t)=L^{2}\left(\mathbb{R}_{-}, \mathrm{d} s\right) \widehat{\otimes} L^{2}((0,1), t \mathrm{~d} t)$. We know that $\left(b_{n}^{[m]}\right)_{n \in \mathbb{N}^{*}}$ is an orthonormal basis of $L^{2}((0,1), t \mathrm{~d} t)$. Then, we construct solutions $\Phi$ and $\Psi$ decomposed in this orthonormal basis. Lemma 13 is an application of the Fredholm alternative after changing the function $g$ to obtain an homogeneous Dirichlet condition at $s=0$. 


\section{Determination of the profiles}

Now, we can start the construction of the Ansatz (15).

Terms in $h^{0} \quad$ The constant terms yield:

$$
\mathcal{L}_{0}^{[m]} \Psi_{0}^{[m]}=\beta_{0}^{[m]} \Psi_{0}^{[m]}, \quad \mathcal{N}_{0}^{[m]} \Phi_{0}^{[m]}=\beta_{0}^{[m]} \Phi_{0}^{[m]}
$$

with boundary conditions (18)-(19) for $j=0$. We choose $\beta_{0}^{[m]}=j_{m, 1}^{2}$. Moreover $\Psi_{0}^{[m]}$ is a tensor product so, $\Psi_{0}^{[m]}=g_{0}^{[m]}(s) b_{1}^{[m]}(t)$. The boundary condition (18) yields : $\Psi_{0}^{[m]}(0, t)=-g_{0}^{[m]}(0) b_{1}^{[m]}(t)$. Lemma 11 gives $g_{0}^{[m]}(0)=0$ and $\Phi_{0}^{[m]} \equiv 0$. The function $g_{0}^{[m]}$ will be determined later.

Terms in $h^{1 / 3} \quad$ Collecting the terms of order $h^{1 / 3}$ we have:

$$
\left(\mathcal{L}_{0}^{[m]}-\beta_{0}^{[m]}\right) \Psi_{1}^{[m]}=\beta_{1}^{[m]} \Psi_{0}^{[m]}-\mathcal{L}_{1}^{[m]} \Psi_{0}^{[m]}=\beta_{1}^{[m]} \Psi_{0}^{[m]}
$$

and

$$
\left(\mathcal{N}_{0}^{[m]}-\beta_{0}^{[m]}\right) \Phi_{1}^{[m]}=\beta_{1}^{[m]} \Phi_{0}^{[m]}-\mathcal{N}_{1}^{[m]} \Phi_{0}^{[m]}=0,
$$

with boundary conditions (18)-(19) for $j=1$. Lemma 12 yields $\beta_{1}^{[m]}=0$ which leads to the following form for the function $\Psi_{1}^{[m]}(s, t)=g_{1}^{[m]}(s) b_{1}^{[m]}(t)$. The boundary condition (18) yields : $\Psi_{1}^{[m]}(0, t)=-g_{1}^{[m]}(0) b_{1}^{[m]}(t)$. Lemma 11 gives $g_{1}^{[m]}(0)=0$ and $\Phi_{1}^{[m]} \equiv 0$.

Terms in $h^{2 / 3} \quad$ Collecting the terms of order $h^{2 / 3}$ we have:

$$
\left(\mathcal{L}_{0}^{[m]}-\beta_{0}^{[m]}\right) \Psi_{2}^{[m]}=\beta_{2}^{[m]} \Psi_{0}^{[m]}-\mathcal{L}_{2}^{[m]} \Psi_{0}^{[m]}
$$

and

$$
\left(\mathcal{N}_{0}^{[m]}-\beta_{0}^{[m]}\right) \Phi_{2}^{[m]}=0
$$

where $\mathcal{L}_{2}^{[m]}=-\partial_{s}^{2}+2 s\left(\frac{1}{t} \partial_{t}\left(t \partial_{t}\right)-\frac{m^{2}}{t^{2}}\right)$ and boundary conditions (18)-(19) for $j=2$. Lemma 12 yields the following equation in the s-variable:

$$
\left\langle\left(\beta_{2}^{[m]}-\mathcal{L}_{2}^{[m]}\right) \Psi_{0}^{[m]}(s, \cdot), b_{1}^{[m]}\right\rangle_{t}=0, \quad s<0
$$

Nevertheless $\Psi_{0}(s, t)=g_{0}^{[m]}(s) b_{1}^{[m]}(t)$, consequently this equation becomes:

$$
\left(-\partial_{s}^{2}-2 j_{m, 1}^{2} s\right) g_{0}^{[m]}(s)=\beta_{2}^{[m]} g_{0}^{[m]}(s), \quad s<0 .
$$

This equation leads to $\beta_{2}^{[m]}=\left(2 j_{m, 1}^{2}\right)^{2 / 3} z_{\mathrm{A}}(n)$ and $g_{0}^{[m]} \equiv a_{n}^{[m]}$.

We deduce that $\left(\mathcal{L}_{0}^{[m]}-\beta_{0}^{[m]}\right) \Psi_{2}^{[m]}=0$ and the $\Psi_{2}^{[m]}$ has the form $\Psi_{2}^{[m]}=g_{2}^{[m]}(s) b_{1}^{[m]}(t)$. The boundary condition (18) yields : $\Psi_{2}^{[m]}(0, t)=-g_{2}^{[m]}(0)(s) b_{1}^{[m]}(t)$. Lemma 11 gives $g_{2}^{[m]}(0)=0$ and $\Phi_{2}^{[m]} \equiv 0$. 
Terms in $h^{3 / 3}$ Collecting the terms of order $h^{3 / 3}$ we have:

$$
\left(\mathcal{L}_{0}^{[m]}-\beta_{0}^{[m]}\right) \Psi_{3}^{[m]}=\beta_{3}^{[m]} \Psi_{0}^{[m]}+\beta_{2}^{[m]} \Psi_{1}^{[m]}-\mathcal{L}_{2}^{[m]} \Psi_{1}^{[m]}
$$

and

$$
\left(\mathcal{N}_{0}^{[m]}-\beta_{0}^{[m]}\right) \Phi_{3}^{[m]}=0
$$

with boundary conditions (18)-(19) for $j=3$. The scalar product with $b_{1}^{[m]}$ (Lemma 12) and then the scalar product with $g_{0}^{[m]}$ (Lemma 13) yield that $\beta_{3}^{[m]}=0$ and $g_{1}^{[m]}$ is parallel to $g_{0}^{[m]}$. We choose $g_{1}^{[m]} \equiv 0$. As a consequence $\Psi_{3}^{[m]}$ has the form $\Psi_{3}^{[m]}(s, t)=g_{3}^{[m]}(s) b_{1}^{[m]}(t)$. Lemma 11 gives $g_{3}^{[m]}(0)=0$ and $\Phi_{3}^{[m]} \equiv 0$.

Terms in $h^{4 / 3} \quad$ Collecting the terms of order $h^{4 / 3}$ we have:

$$
\left(\mathcal{L}_{0}^{[m]}-\beta_{0}^{[m]}\right) \Psi_{4}^{[m]}=\beta_{4}^{[m]} \Psi_{0}^{[m]}+\beta_{2}^{[m]} \Psi_{2}^{[m]}-\mathcal{L}_{4}^{[m]} \Psi_{0}^{[m]}-\mathcal{L}_{2}^{[m]} \Psi_{2}^{[m]}
$$

and

$$
\left(\mathcal{N}_{0}^{[m]}-\beta_{0}^{[m]}\right) \Phi_{4}^{[m]}=0
$$

where

$$
\mathcal{L}_{4}^{[m]}=2 \partial_{t} \partial_{s}-\frac{3 s^{2}}{2}\left(\frac{1}{t} \partial_{t}\left(t \partial_{t}\right)-\frac{m^{2}}{t^{2}}\right)
$$

with boundary conditions (18)-(19) for $j=4$. The scalar product with $b_{1}^{[\mathrm{m}]}$ (Lemma 12) yields an equation for $g_{2}^{[m]}$ and the scalar product with $g_{0}^{[m]}$ (Lemma 13) determined $\beta_{4}^{[m]}$. Thanks to Lemma 12 , $\Psi_{4}^{[m]}$ has the form $\Psi_{4}^{[m]}=\Psi_{4}^{[m] \perp}+g_{4}^{[m]}(s) b_{1}^{[m]}(t)$ with $\Psi_{4}^{[m] \perp}$ which can be nonzero. Lemma 11 yields $g_{4}^{[m]}(0)=0$; moreover $\left\langle\Psi_{4}^{[m] \perp}(0, \cdot), b_{1}^{[m]}\right\rangle_{t}=0$ and we have a solution $\Phi_{4}^{[m]}$ with exponential decay.

Continuation We can construct the further terms by induction along the same lines. This leads to define the quasimodes for $\mathcal{L}^{[m]}(h)$ :

$$
\begin{gathered}
\beta_{h}^{[m, J]}=\sum_{j=0}^{J} \beta_{j}^{[m]} h^{j / 3}, \\
\psi_{h}(x, y)^{[m, J]}=\chi^{\text {lef }}(x) \sum_{j=0}^{J}\left(\Psi_{j}^{[m]}\left(\frac{x}{h^{2 / 3}}, \frac{y}{x+1}\right)+\Phi_{j}^{[m]}\left(\frac{x}{h}, \frac{y}{x+1}\right)\right) h^{j / 3},
\end{gathered}
$$

where $\chi^{\text {lef }}$ is a smooth cut-off function such that:

$$
\chi^{\text {lef }}(x)=1 \quad \text { for } x \in\left(-\frac{1}{2}, 0\right) \quad \text { and } \quad \chi^{\text {lef }}(x)=0 \quad \text { for } x \leq-\frac{3}{4} \text {. }
$$

The spectral theorem yields the conclusion. 


\subsection{Agmon estimates}

On our way to prove Theorem 3, we now state Agmon estimates like for $l_{\mathrm{BO}}^{[m]}(h)$. Let us first notice that, due to Proposition 9, the $N_{0}$ lowest eigenvalues $\lambda$ of $\mathcal{L}^{[m]}(h)$ satisfy:

$$
\left|\lambda-j_{m, 1}^{2}\right| \leq \Gamma_{0} h^{2 / 3}
$$

for some positive constant $\Gamma_{0}$ depending on $N_{0}$.

If we denote by $Q_{h}^{[m]}$ the quadratic form associated with $\mathcal{L}^{[m]}(h)$ we have, for all $\psi \in \operatorname{Dom}\left(Q_{h}^{[m]}\right)$, the following lower bound:

$$
Q_{h}^{[m]}(\psi) \geq \int_{\text {Tri }}\left(h^{2}\left|\partial_{x} \psi\right|^{2}+\frac{j_{m, 1}^{2}}{(x+1)^{2}}|\psi|^{2}\right) y \mathrm{~d} x \mathrm{~d} y .
$$

Thus, the analysis giving Propositions 7 and 8 applies exactly in the same way and we obtain:

Proposition 15 Let $\Gamma_{0}>0$. There exist $h_{0}>0, C_{0}>0$ and $\eta_{0}>0$ such that for $h \in\left(0, h_{0}\right)$ and all eigenpair $(\lambda, \psi)$ of $\mathcal{L}^{[m]}(h)$ satisfying $\left|\lambda-j_{m, 1}^{2}\right| \leq \Gamma_{0} h^{2 / 3}$, we have:

$$
\int_{\text {Tri }} e^{\eta_{0} h^{-1}|x|^{3 / 2}}\left(|\psi|^{2}+\left|h^{2 / 3} \partial_{x} \psi\right|^{2}\right) y \mathrm{~d} x \mathrm{~d} y \leq C_{0}\|\psi\|^{2} .
$$

Proposition 16 Let $\Gamma_{0}>0$. There exist $h_{0}>0, C_{0}>0$ and $\rho_{0} \in\left(0, j_{m, 1}\right)$ such that for $h \in\left(0, h_{0}\right)$ and all eigenpair $(\lambda, \psi)$ of $\mathcal{L}^{[m]}(h)$ satisfying $\left|\lambda-j_{m, 1}^{2}\right| \leq \Gamma_{0} h^{2 / 3}$, we have:

$$
\int_{\text {Tri }}(x+1)^{-\rho_{0} / h}\left(|\psi|^{2}+\left|h \partial_{x} \psi\right|^{2}\right) y \mathrm{~d} x \mathrm{~d} y \leq C_{0}\|\psi\|^{2} .
$$

Remark 17 Propositions 15 and 16 are also verified when $\psi$ is a finite linear combination of eigenfunctions associated with eigenvalues satisfying (25).

\subsection{Approximation of the first eigenfunctions by tensor products}

In this subsection we will work with the operator $\mathcal{L}_{\mathrm{Sq}}^{[m]}(h)$ rather than $\mathcal{L}^{[m]}(h)$. Let us consider the first $N_{0}$ eigenvalues of $\mathcal{L}_{\mathrm{Sq}}^{[m]}(h)$ (shortly denoted by $\lambda_{n}(h)$ ). In each corresponding eigenspace we choose a normalized eigenfunction $\hat{\psi}_{n}$ so that $\left\langle\hat{\psi}_{n}, \hat{\psi}_{p}\right\rangle=0$ if $n \neq p$. We introduce:

$$
\widehat{\mathcal{S}}_{N_{0}}(h)=\operatorname{span}\left(\hat{\psi}_{1}, \ldots, \hat{\psi}_{N_{0}}\right) \text {. }
$$

Let us define $Q_{\mathrm{Sq}}^{0,[m]}$ the following quadratic form:

$$
Q_{\mathrm{Sq}}^{0,[m]}(\hat{\psi})=\int_{\mathrm{Sq}}\left(\left|\partial_{t} \hat{\psi}\right|^{2}+\frac{m^{2}}{t^{2}}|\hat{\psi}|^{2}-j_{m, 1}^{2}|\hat{\psi}|^{2}\right) t(u+1)^{2} \mathrm{~d} u \mathrm{~d} t
$$

associated with the operator $\mathcal{L}_{\mathrm{Sq}}^{0,[m]}=\mathrm{Id}_{u} \otimes\left(-\frac{1}{t} \partial_{t}\left(t \partial_{t}\right)+\frac{m^{2}}{t^{2}}-j_{m, 1}^{2}\right)$ on $L^{2}\left(\mathrm{Sq}, t(u+1)^{2} \mathrm{~d} u \mathrm{~d} t\right)$. We consider the Feshbach-Grushin projection on the eigenspace associated with the eigenvalue 0 of $-\frac{1}{t} \partial_{t}\left(t \partial_{t}\right)+\frac{m^{2}}{t^{2}}-j_{m, 1}^{2}$ :

$$
\Pi_{1}^{[m]} \hat{\psi}(u, t)=\left\langle\hat{\psi}(u, \cdot), b_{1}^{[m]}\right\rangle_{t} b_{1}^{[m]}(t)
$$

We can now state a first approximation result: 
Proposition 18 There exist $h_{0}>0$ and $C>0$ such that for $h \in\left(0, h_{0}\right)$ and all $\hat{\psi} \in \widehat{\mathcal{S}}_{N_{0}}(h)$ :

$$
0 \leq Q_{\mathrm{Sq}}^{0,[m]}(\hat{\psi}) \leq C h^{2 / 3}\|\hat{\psi}\|^{2}
$$

and

$$
\left\|\left(I d-\Pi_{1}^{[m]}\right) \hat{\psi}\right\|+\left\|\frac{1}{t}\left(I d-\Pi_{1}^{[m]}\right) \hat{\psi}\right\|+\left\|\partial_{t}\left(I d-\Pi_{1}^{[m]}\right) \hat{\psi}\right\| \leq C h^{1 / 3}\|\hat{\psi}\| .
$$

Moreover we have, $\Pi_{1}^{[m]}: \widehat{\mathcal{S}}_{N_{0}}(h) \rightarrow \Pi_{1}^{[m]}\left(\widehat{\mathcal{S}}_{N_{0}}(h)\right)$ is an isomorphism.

Proof: If $\hat{\psi}=\hat{\psi}_{n}$ we have:

$$
Q_{\mathrm{Sq}, h}^{[m]}\left(\hat{\psi}_{n}\right)=\lambda_{n}\left\|\hat{\psi}_{n}\right\|^{2}
$$

From this we infer:

$$
Q_{\mathrm{Sq}, h}^{[m]}\left(\hat{\psi}_{n}\right) \leq\left(j_{m, 1}^{2}+C h^{2 / 3}\right)\left\|\hat{\psi}_{n}\right\|^{2} .
$$

The orthogonality of the $\hat{\psi}_{n}$ (in $L^{2}$ and for the quadratic form) allows to extend this inequality to $\hat{\psi} \in \widehat{\mathcal{S}}_{N_{0}}(h)$ :

$$
Q_{\mathrm{Sq}}^{0,[m]}(\hat{\psi}) \leq C h^{2 / 3}\|\hat{\psi}\|^{2}
$$

Moreover $\Pi_{1}^{[m]} \hat{\psi}$ being in the kernel of $\mathcal{L}_{\mathrm{Sq}}^{0,[m]}$, we have:

$$
\begin{aligned}
Q_{\mathrm{Sq}}^{0,[m]}(\hat{\psi}) & \left.=\left\langle\mathcal{L}_{\mathrm{Sq}}^{0,[m]}\left(\Pi_{1}^{[m]} \hat{\psi}+\left(I d-\Pi_{1}^{[m]}\right) \hat{\psi}\right), \hat{\psi}\right)\right\rangle \\
& =\left\langle\mathcal{L}_{\mathrm{Sq}}^{0,[m]}\left(I d-\Pi_{1}^{[m]}\right) \hat{\psi}, \hat{\psi}\right\rangle \\
& =Q_{\mathrm{Sq}}^{0,[m]}\left(\left(I d-\Pi_{1}^{[m]}\right) \hat{\psi}\right) .
\end{aligned}
$$

If $\mu_{2}$ denotes the second eigenvalue of the one dimensional operator $-\frac{1}{t} \partial_{t}\left(t \partial_{t}\right)+\frac{m^{2}}{t^{2}}-j_{m, 1}^{2}$ we have, for all $u \in(-1,0)$, thanks to the min-max principle:

$\int_{0}^{1}\left|\partial_{t}\left(\left(I d-\Pi_{1}^{[m]}\right) \hat{\psi}\right)\right|^{2}+\frac{m^{2}}{t^{2}}\left|\left(I d-\Pi_{1}^{[m]}\right) \hat{\psi}\right|^{2}-j_{m, 1}^{2}\left|\left(I d-\Pi_{1}^{[m]}\right) \hat{\psi}\right|^{2} t \mathrm{~d} t \geq \mu_{2} \int_{0}^{1}\left|\left(I d-\Pi_{1}^{[m]}\right) \hat{\psi}\right|^{2} t \mathrm{~d} t$.

Multiplying by $(u+1)^{2}$ and taking the integral over $u \in(-1,0)$, we obtain:

$$
Q_{\mathrm{Sq}}^{0,[m]}\left(\left(I d-\Pi_{1}^{[m]}\right) \hat{\psi}\right) \geq \mu_{2}\left\|\left(I d-\Pi_{1}^{[m]}\right) \hat{\psi}\right\|^{2} .
$$

We deduce that:

$$
0 \leq Q_{\operatorname{Rec}}^{0,[m]}(\hat{\psi}) \leq C h^{2 / 3}\|\hat{\psi}\|^{2} \quad ; \quad\left\|\frac{1}{t}\left(I d-\Pi_{1}^{[m]}\right) \hat{\psi}\right\|+\left\|\partial_{t}\left(I d-\Pi_{1}^{[m]}\right) \hat{\psi}\right\| \leq C h^{1 / 3}\|\hat{\psi}\| .
$$

We also have:

$$
\left\|\left(I d-\Pi_{1}^{[m]}\right) \hat{\psi}\right\| \leq C h^{1 / 3}\|\hat{\psi}\|
$$

which yields Proposition 18. 


\subsection{Reduction to the Born-Oppenheimer approximation}

The aim of this subsection is to prove Theorem 3 using the projections of the true eigenfunctions $\left(\Pi_{1}^{[m]} \hat{\psi}_{n}\right)$ as test functions for the quadratic form of the Airy operator. It justifies that $l_{\mathrm{BO}}^{[m]}(h)$ is a good approximation of $\mathcal{L}^{[m]}(h)$. Let us consider $\hat{\psi} \in \widehat{S}_{N_{0}}(h)$, we will need a few lemmas to estimate the quadratic form of the Airy operator tested on $\Pi_{1}^{[m]} \hat{\psi}$. The first lemma is an estimate in the triangle Tri; we let $\hat{\psi}(u, t)=\psi(x, y)$ and we consider the space $S_{N_{0}}(h)$

$$
S_{N_{0}}(h):=\operatorname{span}\left(\psi_{1}, \ldots, \psi_{N_{0}}\right) .
$$

Then we have the

Lemma 19 For all $\psi \in S_{N_{0}}(h)$ and for all $k \in \mathbb{N}$, there exist $h_{0}>0$ and $C>0$ such that we have, for $h \in\left(0, h_{0}\right)$ :

$$
\int_{\operatorname{Tri}}(x+1)^{-k}\left|\partial_{y} \psi\right|^{2} y \mathrm{~d} x \mathrm{~d} y \leq C\|\psi\|^{2}
$$

Proof: First let $\psi=\psi_{j}$ for some $j \in\left\{1, \ldots, N_{0}\right\}$. It satisfies the equation:

$$
\left(-h^{2} \partial_{x}^{2}-\frac{1}{y} \partial_{y}\left(y \partial_{y}\right)+\frac{m^{2}}{y^{2}}\right) \psi_{j}=\lambda_{j}(h) \psi_{j}
$$

Multiplying by $(x+1)^{-k}$, taking the scalar product with $\psi_{j}$ and integrating by parts we find:

$$
\int_{\text {Tri }}(x+1)^{-k}\left|\partial_{y} \psi_{j}\right|^{2} y \mathrm{~d} x \mathrm{~d} y \leq C \int_{\text {Tri }}(x+1)^{-k}\left(\left|\psi_{j}\right|^{2}+h^{2}(x+1)^{-1}\left|\psi_{j}\right|\left|\partial_{x} \psi_{j}\right|\right) y \mathrm{~d} x \mathrm{~d} y .
$$

Using the Agmon estimates of Proposition 16 with $\rho_{0} \geq k+1$ we deduce the lemma for $\psi=\psi_{j}$. For $\psi \in S_{N_{0}}(h)$, we proceed as explained in Remark 17 .

We can now prove:

Lemma 20 Let $\hat{\psi}$ be in $\widehat{S}_{N_{0}}(h)$. There exists $h_{0}>0$ and $C>0$ such that for all $h \in\left(0, h_{0}\right)$

$$
\left|h^{2} \int_{\text {Sq }} \frac{1}{(u+1)^{2}}\left(\partial_{u} \hat{\psi}\right)\left(\partial_{t} \hat{\psi}\right) t(u+1)^{2} \mathrm{~d} t \mathrm{~d} u\right| \leq C h^{4 / 3}\|\hat{\psi}\|^{2} .
$$

Proof: Thanks to the Cauchy-Schwartz inequality we have:

$\left|h^{2} \int_{\mathrm{Sq}} \frac{1}{(u+1)^{2}}\left(\partial_{u} \hat{\psi}\right)\left(\partial_{t} \hat{\psi}\right) t(u+1)^{2} \mathrm{~d} t \mathrm{~d} u\right|^{2} \leq h^{4} \int_{\mathrm{Sq}}\left|\partial_{u} \hat{\psi}\right|^{2}(u+1)^{2} t \mathrm{~d} t \mathrm{~d} u \int_{\mathrm{Sq}} \frac{1}{(u+1)^{4}}\left|\partial_{t} \hat{\psi}\right|^{2} t(u+1)^{2} \mathrm{~d} t \mathrm{~d} u$.

In the original coordinates on the meridian domain Tri we have:

$$
\int_{\mathrm{Sq}} \frac{1}{(u+1)^{4}}\left|\partial_{t} \hat{\psi}\right|^{2} t(u+1)^{2} \mathrm{~d} t \mathrm{~d} u=\int_{\text {Tri }}(x+1)^{-4}\left|\partial_{y} \psi\right|^{2} y \mathrm{~d} x \mathrm{~d} y .
$$

Combining Lemma 19 with this equality we obtain

$$
\int_{\mathrm{Sq}} \frac{1}{(u+1)^{4}}\left|\partial_{t} \hat{\psi}\right|^{2} t(u+1)^{2} \mathrm{~d} t \mathrm{~d} u \leq C_{1}\|\hat{\psi}\|^{2}
$$


for some $C_{1}>0$. Using Proposition 15 expressed in the Square Sq coordinates, there exists $C_{2}>0$ such that:

$$
\int_{\text {Sq }}\left|\partial_{u} \hat{\psi}-\frac{1}{(u+1)^{2}} \partial_{t} \hat{\psi}\right|^{2} t(u+1)^{2} \mathrm{~d} t \mathrm{~d} u \leq C_{2} h^{-4 / 3}\|\hat{\psi}\|^{2} .
$$

For some $C_{3}>0$, equation (28) yields

$$
\int_{\mathrm{Sq}}\left|\partial_{u} \hat{\psi}\right|^{2} t(u+1)^{2} \mathrm{~d} t \mathrm{~d} u \leq C_{3} h^{-4 / 3}\|\hat{\psi}\|^{2}
$$

which achieves the proof of the lemma.

To have estimates in $L^{2}(\mathrm{Sq}, t \mathrm{~d} t \mathrm{~d} u)$ instead of $L^{2}\left(\mathrm{Sq}, t(u+1)^{2} \mathrm{~d} t \mathrm{~d} u\right)$ we will need the

Lemma 21 Let $\hat{\psi}$ be in $\widehat{S}_{N_{0}}(h)$. There exists $h_{0}>0$ and $C>0$ such that for all $h \in\left(0, h_{0}\right)$

$$
\left.\left|h^{2} \int_{\mathrm{Sq}}\right| \partial_{u} \hat{\psi}\right|^{2} u t \mathrm{~d} t \mathrm{~d} u\left|\leq C h^{4 / 3}\right|\left|\hat{\psi}\left\|^{2} ;\left.\quad\left|\int_{\mathrm{Sq}}\right| u|| \hat{\psi}\right|^{2} u t \mathrm{~d} t \mathrm{~d} u \mid \leq C h^{4 / 3}\right\| \hat{\psi} \|^{2} .\right.
$$

Proof: We express each integral in the meridian domain Tri and we use the Agmon estimate of Proposition 15 for getting the lemma.

We can now prove the

Proposition 22 Let $\hat{\psi} \in \widehat{S}_{N_{0}}(h)$. There exists $h_{0}>0$ and $C>0$ such that for all $h \in\left(0, h_{0}\right)$

$$
\int_{\mathrm{Sq}}\left(h^{2}\left|\partial_{u} \hat{\psi}\right|^{2}+j_{m, 1}^{2}|u||\hat{\psi}|^{2}\right) t \mathrm{~d} t \mathrm{~d} u \leq\left(\lambda_{N_{0}}(h)-j_{m, 1}^{2}\right)\|\hat{\psi}\|^{2}+C h^{4 / 3}\|\hat{\psi}\|^{2} .
$$

Proof: Let us consider $\psi \in S_{N_{0}}(h)$. As the $\left(\psi_{i}\right)_{i \in\left\{1, \ldots, N_{0}\right\}}$ are orthogonal, we have:

$$
Q_{h}^{[m]}(\psi) \leq \lambda_{N_{0}}(h)\|\psi\|^{2} .
$$

Equation (26) leads to

$$
\int_{\text {Tri }} h^{2}\left|\partial_{x} \psi\right|^{2}+\frac{j_{m, 1}^{2}}{(x+1)^{2}}|\psi|^{2} y \mathrm{~d} x \mathrm{~d} y \leq \lambda_{N_{0}}(h)\|\psi\|^{2}
$$

Using the convexity of the function $\left(x \mapsto \frac{1}{(x+1)^{2}}\right)$ we get

$$
\int_{\text {Tri }}\left(h^{2}\left|\partial_{x} \psi\right|^{2}+j_{m, 1}^{2}|x||\psi|^{2}\right) y \mathrm{~d} x \mathrm{~d} y \leq\left(\lambda_{N_{0}}(h)-j_{m, 1}^{2}\right)\|\psi\|^{2} .
$$

Performing the change of variable (11) and thanks to Lemmas 20 and 21, we obtain in the square Sq:

$$
\int_{\mathrm{Sq}}\left(h^{2}\left|\partial_{u} \hat{\psi}\right|^{2}+j_{m, 1}^{2}|u||\hat{\psi}|^{2}\right) t \mathrm{~d} t \mathrm{~d} u \leq\left(\lambda_{N_{0}}(h)-j_{m, 1}^{2}\right)\|\hat{\psi}\|^{2}+C h^{4 / 3}\|\hat{\psi}\|^{2},
$$

which ends the proof of the proposition. 
Proof of Theorem 3 The inequality of Proposition 22 can be written as

$$
Q_{\mathrm{A}, h}^{[m]}(\hat{\psi}) \leq\left(\lambda_{N_{0}}(h)-j_{m, 1}^{2}\right)\|\hat{\psi}\|^{2}+C h^{4 / 3}\|\hat{\psi}\|^{2}
$$

where $Q_{\mathrm{A}, h}^{[m]}$ is the positive quadratic form associated with an Airy operator defined by

$$
Q_{\mathrm{A}, h}^{[m]}(\hat{\psi}):=\int_{\mathrm{Sq}}\left(h^{2}\left|\partial_{u} \hat{\psi}\right|^{2}+j_{m, 1}^{2}|u||\hat{\psi}|^{2}\right) t \mathrm{~d} t \mathrm{~d} u .
$$

Proposition 18 and (25) give

$$
Q_{\mathrm{A}, h}^{[m]}(\hat{\psi}) \leq\left(\lambda_{N_{0}}(h)-j_{m, 1}^{2}\right)\left\|\Pi_{1}^{[m]} \hat{\psi}\right\|_{L^{2}(\mathrm{Sq}, t \mathrm{~d} t \mathrm{~d} u)}^{2}+C h^{4 / 3}\left\|\Pi_{1}^{[m]} \hat{\psi}\right\|_{L^{2}(\mathrm{Sq}, t \mathrm{~d} t \mathrm{~d} u)}^{2} .
$$

Moreover we obtain

$$
Q_{\mathrm{A}, h}^{[m]}(\hat{\psi})=Q_{\mathrm{A}, h}^{[m]}\left(\Pi_{1}^{[m]} \hat{\psi}\right)+Q_{\mathrm{A}, h}^{[m]}\left(\left(I d-\Pi_{1}^{[m]}\right) \hat{\psi}\right)+2 b_{\mathrm{A}, h}^{[m]}\left(\Pi_{1}^{[m]} \hat{\psi},\left(I d-\Pi_{1}^{[m]}\right) \hat{\psi}\right),
$$

where $b_{\mathrm{A}, h}^{[m]}$ is the bilinear form associated with $Q_{\mathrm{A}, h}^{[m]}$.

We remark that

$$
b_{\mathrm{A}, h}^{[m]}\left(\Pi_{1}^{[m]} \hat{\psi},\left(I d-\Pi_{1}^{[m]}\right) \hat{\psi}\right)=\int_{u}\left\langle\Pi_{1}^{[m]}\left(\left(-h^{2} \partial_{u}^{2}+j_{m, 1}^{2}|u|\right) \hat{\psi}\right),\left(I d-\Pi_{1}^{[m]}\right) \hat{\psi}\right\rangle_{t} \mathrm{~d} u=0 .
$$

Finally we have

$$
Q_{\mathrm{A}, h}^{[m]}\left(\Pi_{1}^{[m]} \hat{\psi}\right) \leq\left(\lambda_{N_{0}}(h)-j_{m, 1}^{2}\right)\left\|\Pi_{1}^{[m]} \hat{\psi}\right\|_{L^{2}(\mathrm{Sq}, t \mathrm{~d} t \mathrm{~d} u)}^{2}+C h^{4 / 3}\left\|\Pi_{1}^{[m]} \hat{\psi}\right\|_{L^{2}(\mathrm{Sq}, t \mathrm{~d} t \mathrm{~d} u)}^{2} .
$$

Let us denote by $\pi_{1}^{[m]} \hat{\psi}:=\left\langle\Pi_{1}^{[m]} \hat{\psi}, b_{1}^{[m]}\right\rangle_{t}$, it is a funtion in the only $u$-variable and we have in one dimension:

$q_{\mathrm{A}, h}^{[m]}\left(\pi_{1}^{[m]} \hat{\psi}\right):=\int_{-1}^{0} h^{2}\left|\partial_{u} \pi_{1}^{[m]} \hat{\psi}\right|^{2}+j_{m, 1}^{2}|u|\left|\pi_{1}^{[m]} \hat{\psi}\right|^{2} \mathrm{~d} u \leq\left(\lambda_{N_{0}}(h)-j_{m, 1}^{2}\right)\left\|\pi_{1}^{[m]} \hat{\psi}\right\|^{2}+C h^{4 / 3}\left\|\pi_{1}^{[m]} \hat{\psi}\right\|^{2}$, where the norms $\left\|\pi_{1}^{[m]} \hat{\psi}\right\|$ are taken on $L^{2}((-1,0), \mathrm{d} u)$.

Let us consider a smooth cut-off function $\chi$ such that:

$$
\chi(u)=1 \quad \text { for } u \in\left(-\frac{1}{2}, 0\right) \quad \text { and } \quad \chi(u)=0 \quad \text { for } u \leq-\frac{3}{4} .
$$

Proposition 15 gives

$$
q_{\mathrm{A}, h}^{[m]}\left(\chi \pi_{1}^{[m]} \hat{\psi}\right)+\mathcal{O}\left(h^{\infty}\right)\left\|\pi_{1}^{[m]} \hat{\psi}\right\|^{2} \leq q_{\mathrm{A}, h}^{[m]}\left(\pi_{1}^{[m]} \hat{\psi}\right), \quad \text { and }\left\|\pi_{1}^{[m]} \hat{\psi}\right\|^{2}=\left(1+\mathcal{O}\left(h^{\infty}\right)\right)\left\|\chi \pi_{1}^{[m]} \hat{\psi}\right\|^{2} .
$$

So it holds

$$
q_{\mathrm{A}, h}^{[m]}\left(\chi \pi_{1}^{[m]} \hat{\psi}\right) \leq\left(\lambda_{N_{0}}(h)-j_{m, 1}^{2}\right)\left\|\chi \pi_{1}^{[m]} \hat{\psi}\right\|^{2}+C h^{4 / 3}\left\|\chi \pi_{1}^{[m]} \hat{\psi}\right\|^{2}
$$

Then, we consider $\widehat{s}_{N_{0}}(h):=\operatorname{span}\left(\pi_{1}^{[m]} \hat{\psi}_{1}, \ldots, \pi_{1}^{[m]} \hat{\psi}_{N_{0}}\right)$ and apply the min-max principal to the $N_{0}$ dimensional space $\chi \widehat{s}_{N_{0}}(h)$ which yields

$$
j_{m, 1}^{2}+\left(2 j_{m, 1}^{2}\right)^{2 / 3} z_{\mathrm{A}}\left(N_{0}\right) h^{2 / 3} \leq \lambda_{N_{0}}(h)+C h^{4 / 3} .
$$

Jointly with Proposition 9 this finishes the proof of Theorem 3. 


\section{A Shape of the eigenfunctions in the semi-classical limit}

To illustrate some properties of the eigenfunctions we compute some of them. These computations are performed in the scaled meridian domain Tri for the scaled operator $\mathcal{L}^{[m]}(\tan \theta)$ defined in (6).

Figure 7 pictures the dominant term in the construction (23) : it is almost a tensor product of the Airy function of first kind and the Bessel function of first kind (respectively along the $\mathrm{X}$-axis and Y-axis). In addition, the eigenfunctions are localized near the right boundary and away from the left corner. This matches the Agmon estimates of Propositions 15 and 16.

Figure 8 shows the localization for increasing values of $m$. As explained in Remark 2 for nonzero $m$, there is a Dirichlet boundary condition along the $\mathrm{X}$-axis which induces a repulsion from this axis. The eigenfunction is localized in the top right corner. We observe that this repulsion increases with the value of $m$. We can interpret this phenomenon: the shape of the $m^{\text {th }}$ Bessel function of first kind determines the behavior along the Y-axis.

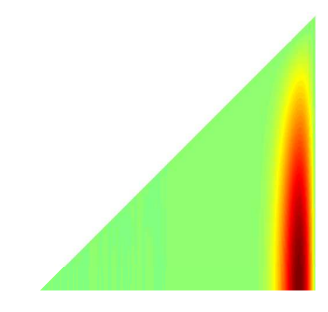

$\mu_{1}^{[0]}(\theta)=7.199103$

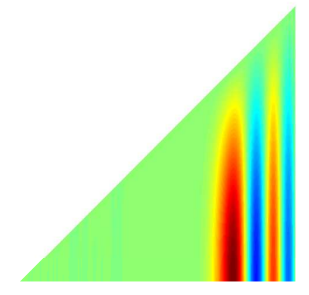

$\mu_{4}^{[0]}(\theta)=10.631834$

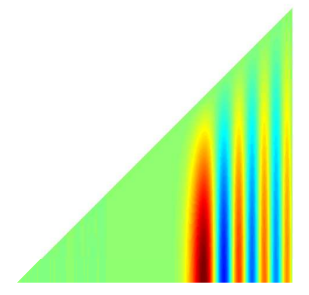

$\mu_{7}^{[0]}(\theta)=13.863783$

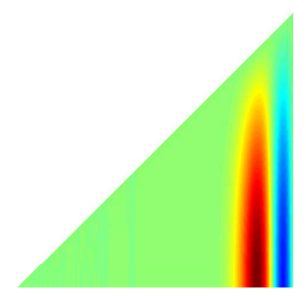

$\mu_{2}^{[0]}(\theta)=8.425123$

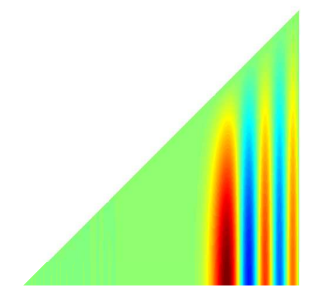

$\mu_{5}^{[0]}(\theta)=11.706005$

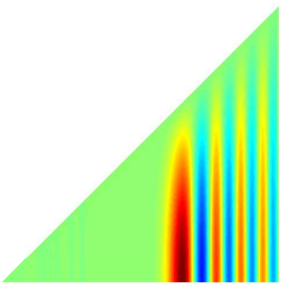

$\mu_{8}^{[0]}(\theta)=14.958588$ $\mu_{3}^{[0]}(\theta)=9.546450$

$\mu_{6}^{[0]}(\theta)=12.781028$
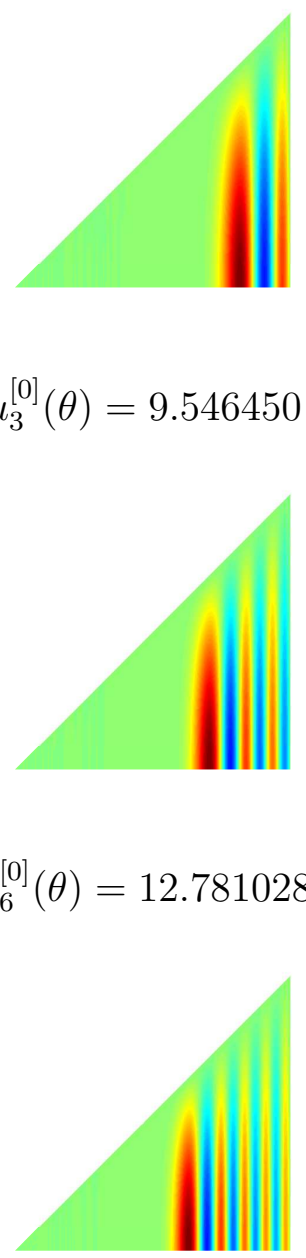

$\mu_{9}^{[0]}(\theta)=16.068338$

Figure 7: Computation for $\theta=0.0226 * \pi / 2 \sim 2^{\circ}$. Numerical values of the nine first eigenvalues for $m=0$. Plots of the associated eigenfunctions in the domain Tri. 


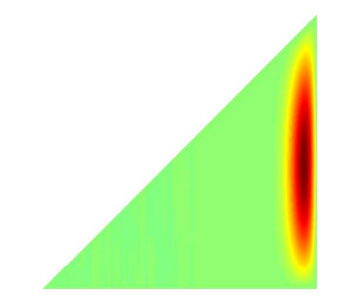

$\mu_{1}^{[1]}(\theta)=17.255710$

$\mu_{1}^{[2]}(\theta)=30.134666$

$\mu_{1}^{[3]}(\theta)=45.692334$ $\mu_{2}^{[1]}(\theta)=19.400598$

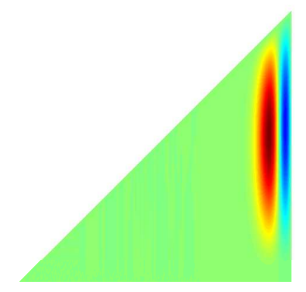

$\mu_{2}^{[2]}(\theta)=33.208960$
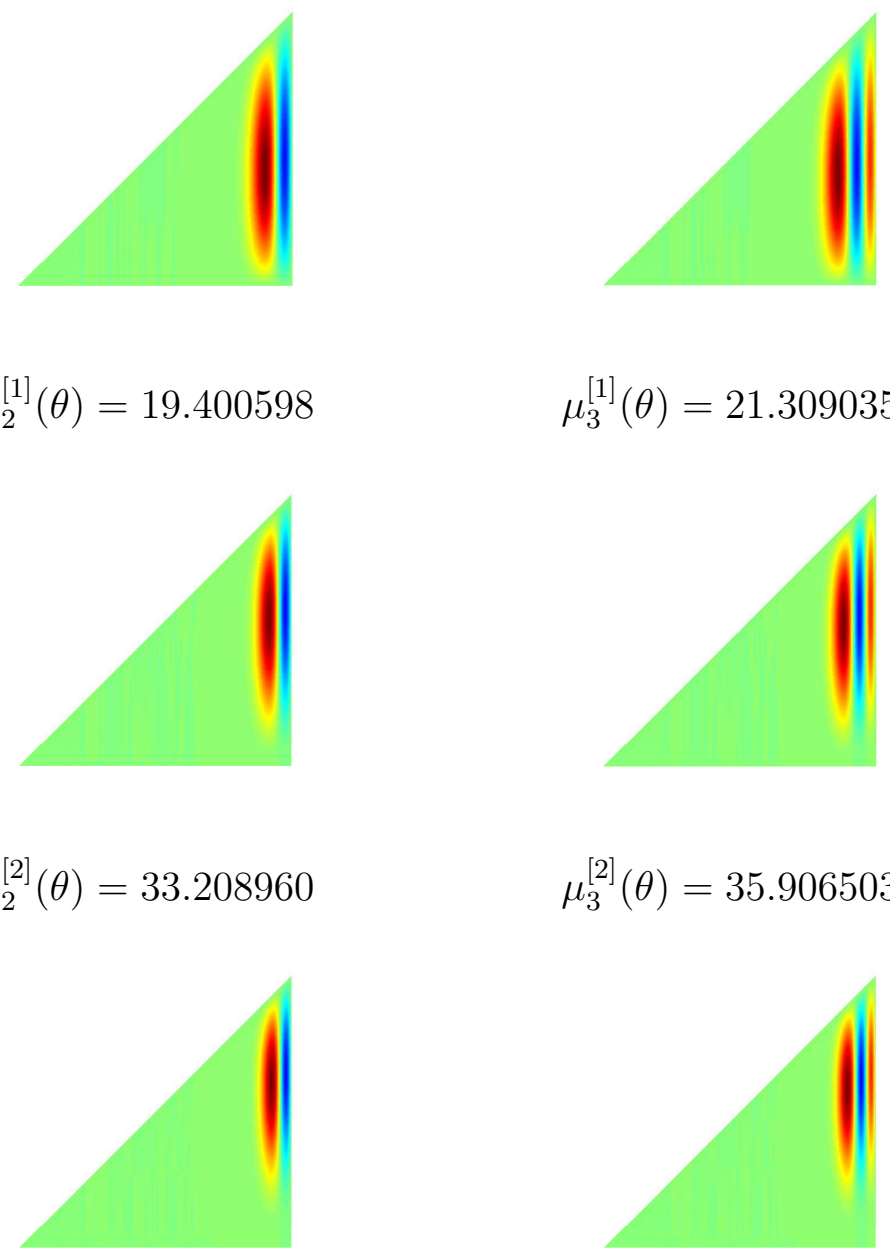

$\mu_{3}^{[1]}(\theta)=21.309035$

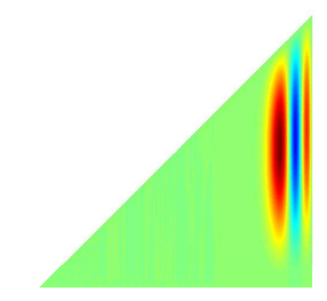

$\mu_{3}^{[2]}(\theta)=35.906503$

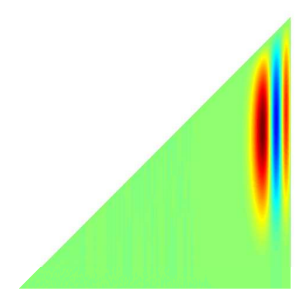

$\mu_{2}^{[3]}(\theta)=49.719970$

$\mu_{3}^{[3]}(\theta)=53.222789$

Figure 8: Computation for $\theta=0.0226 * \pi / 2 \sim 2^{\circ}$. Numerical values of the three first eigenvalues for $m=1, m=2$ and $m=3$. Plots of the associated eigenfunctions in the meridian domain Tri.

Acknowledgements I would like to thank Monique Dauge and Nicolas Raymond for all their remarks and advice on this subject. My thanks also go to Yvon Lafranche who taught me how to use Melina++ ([21]).

\section{References}

[1] S. Agmon. Lectures on exponential decay of solutions of second-order elliptic equations: bounds on eigenfunctions of $N$-body Schrödinger operators, volume 29 of Mathematical Notes. Princeton University Press, Princeton, NJ, 1982.

[2] S. Agmon. Bounds on exponential decay of eigenfunctions of Schrödinger operators. In Schrödinger operators (Como, 1984), volume 1159 of Lecture Notes in Math., pages 1-38. Springer, Berlin, 1985. 
[3] Y. Avishai, D. Bessis, B. G. Giraud, and G. Mantica. Quantum bound states in open geometries. Phys. Rev. $B, 44: 8028-8034$, Oct 1991.

[4] C. Bernardi, M. Dauge, and Y. Maday. Spectral methods for axisymmetric domains, volume 3 of Series in Applied Mathematics (Paris). Gauthier-Villars, Éditions Scientifiques et Médicales Elsevier, Paris, 1999. Numerical algorithms and tests due to Mejdi Azaïez.

[5] J. P. Carini, J. T. Londergan, K. Mullen, and D. P. Murdock. Multiple bound states in sharply bent waveguides. Phys. Rev. B, 48:4503-4515, Aug 1993.

[6] G. Carron, P. Exner, and D. Krejčǐřík. Topologically nontrivial quantum layers. J. Math. Phys., 45(2):774$784,2004$.

[7] B. Chenaud, P. Duclos, P. Freitas, and D. Krejčiřík. Geometrically induced discrete spectrum in curved tubes. Differential Geom. Appl., 23(2):95-105, 2005.

[8] J. Combes, P. Duclos, and R. Seiler. The born-oppenheimer approximation. In G. Velo and A. Wightman, editors, Rigorous Atomic and Molecular Physics, volume 74 of NATO Advanced Study Institutes Series, pages 185-213. Springer US, 1981.

[9] H. L. Cycon, R. G. Froese, W. Kirsch, and B. Simon. Schrödinger operators with application to quantum mechanics and global geometry. Texts and Monographs in Physics. Springer-Verlag, Berlin, study edition, 1987.

[10] M. Dauge, Y. Lafranche, and N. Raymond. Quantum waveguides with corners. ESAIM: Proceedings, 35:14-45, 2012.

[11] M. Dauge and N. Raymond. Plane waveguides with corners in the small angle limit. Journal of Mathematical Physics, 53(12):123529, 2012.

[12] M. Dimassi and J. Sjöstrand. Spectral asymptotics in the semi-classical limit, volume 268 of London Mathematical Society Lecture Note Series. Cambridge University Press, Cambridge, 1999.

[13] P. Duclos and P. Exner. Curvature-induced bound states in quantum waveguides in two and three dimensions. Rev. Math. Phys., 7(1):73-102, 1995.

[14] P. Exner and M. Tater. Spectrum of Dirichlet Laplacian in a conical layer. J. Phys. A, 43(47):474023, 11, 2010.

[15] P. Exner, P. Šeba, and P. Št'oviček. On existence of a bound state in an L-shaped waveguide. Czechoslovak Journal of Physics, 39:1181-1191, 1989.

[16] S. Fournais and B. Helffer. Spectral methods in surface superconductivity. Progress in Nonlinear Differential Equations and their Applications, 77. Birkhäuser Boston Inc., Boston, MA, 2010.

[17] P. Freitas. Precise bounds and asymptotics for the first Dirichlet eigenvalue of triangles and rhombi. $J$. Funct. Anal., 251(1):376-398, 2007.

[18] B. Helffer. Semi-classical analysis for the Schrödinger operator and applications, volume 1336 of Lecture Notes in Mathematics. Springer-Verlag, Berlin, 1988.

[19] B. Helffer and J. Sjöstrand. Multiple wells in the semiclassical limit. I. Comm. Partial Differential Equations, 9(4):337-408, 1984. 
[20] M. Klein, A. Martinez, R. Seiler, and X. P. Wang. On the Born-Oppenheimer expansion for polyatomic molecules. Comm. Math. Phys., 143(3):607-639, 1992.

[21] Y. Lafranche and D. Martin. MÉLINA++, bibliothèque de calculs éléments finis. http://anum-maths.univ-rennes1.fr/melina/, 2012.

[22] A. Martinez. Développements asymptotiques et effet tunnel dans l'approximation de Born-Oppenheimer. Ann. Inst. H. Poincaré Phys. Théor., 50(3):239-257, 1989.

[23] M. Reed and B. Simon. Methods of modern mathematical physics. IV. Analysis of operators. Academic Press, New York, 1978.

[24] B. Simon. Semiclassical analysis of low lying eigenvalues. I. Nondegenerate minima: asymptotic expansions. Ann. Inst. H. Poincaré Sect. A (N.S.), 38(3):295-308, 1983. 